\title{
THE ANTHROPOLOGICAL ASSESSMENT OF THE LATE ROMAN CEMETERY AT SOMOGYSZIL-DÖGKÚTI DÜLŐ
}

\author{
SÁNDOR ÉVINGER - ZSOLT BERNERT
}

\author{
Department of Anthropology \\ Hungarian Natural History Museum \\ 2-6 Ludovika tér, H-1083 Budapest, Hungary \\ evinger.sandor@nhmus.hu \\ bernert.zsolt@nhmus.hu
}

\begin{abstract}
The results of a general anthropological examination of 140 individuals from a late Roman period cemetery at Somogyszil-Dögkút site are presented in this paper. The population had a more or less balanced sex ratio, lived a fundamentally peaceful life suggested by the low frequencey of bone injuries, and according to their morphoscopic traits, they all belonged to the Caucasoid group. Based on the biological distances calculated from selected linear measurements of male crania, the population of Somogyszil-Dögkút proved to be quite similar to several other late Roman period cemeteries in Transdanubia, as well as to some local Avar period series. This raises the possibility of a significant local continuity between the late Roman and late Avar period on this territory, however other potential explanations cannot be ruled out. Some anthropological characteristics of the human skeletal material unearthed from graves oriented differently than the cemetery's norm suggest the presence of immigrants in the community. Their biological background cannot be traced from the present data, however a few skeletal evidence proposes the probability of a Sarmatian origin.
\end{abstract}

Keywords: Somogyszil, late Roman period, anthropology, biological distance

\section{INTRODUCTION}

The first finds of a late Roman period cemetery at Somogyszil-Dögkút was found in 1964, and until the end of 1968 altogether 148 graves had been excavated by the archaeologist Balázs Draveczky. ${ }^{1}$ A full archaeological publication of the cemetery was given first by Alice Sz. Burger ${ }^{2}$ with some basic anthropological information about the human skeletal remains provided by Tibor Tóth. However, recently both the archaeological and anthropological material was put to a re-assessment. ${ }^{3}$ In this paper a more detailed version of the results of the anthropological examination is presented.

\section{MATERIAL AND METHODS}

The human skeletal material of Somogyszil-Dögkúti dülö site is housed in the Department of Anthropology of the Hungarian Natural History Museum under the inventory numbers 68.126.1. - 68.126.124. and 68.150.1. 68.150.17. The general preservation of the bones are quite mediocre on average with many of them being strongly incomplete and/or badly preserved.

For scoring morphological sex, altogether 21 anatomical characteristics indicating sexual dimorphism were used. $^{4}$

${ }^{1}$ DraVeCZKY 1965; DraVeCZKy 1966; DraVeCZKy 1967.

${ }^{2}$ BURGER 1979.

\footnotetext{
${ }^{3}$ HoRváth et al. 2018.

${ }^{4}$ ÉRY et al. 1963; ÉRY 1992.
} 
The biological age estimation of children was based on tooth eruption ${ }^{5}$ and on the maximum length of longbones. ${ }^{6}$ In the case of juveniles, the union of certain ossification centres was checked. ${ }^{7}$ The biological age of adults was estimated on the basis of the surface alterations of the pubic symphysis, ${ }^{8}$ the ossification of cranial sutures, ${ }^{9}$ the alterations of the sternal rib ends, ${ }^{10}$ the wear of permanent dentition ${ }^{11}$ and on the root transparency of teeth. ${ }^{12}$

Cranial and longbone measurements and indices were taken according to the work of R. Martin and K. Saller. ${ }^{13}$ Cranial indices were categorized into classes based on the recommendations of V. P. Alekseyev and G. F. Debetz. ${ }^{14}$ Cranial capacity was calculated with the method of A. Lee and K. Pearson. ${ }^{15}$ Using the mean standard deviations of cranial measurements and indices given by V. P. Alekseyev and G. F. Debetz sigma ratios (S.R.) ${ }^{16}$ were calculated which offer basic information about the relative measure of variance of these traits. This way, they tell whether the examined population was more heterogeneous or homogeneous than a theoretical natural population based on their selected measurements and indices.

Stature was calculated using the formula proposed by T. Sjøvold ${ }^{17}$ that controls for all geographic areas and for both sexes using the femur. If the femur was not measurable, the tibia was used instead.

Mortality tables for the demographic analysis were created based on the works of D. H. Ubelaker ${ }^{18}$ and K. Éry ${ }^{19}$ using the Excel software package created by Zs. Bernert. ${ }^{20}$ For the „lack of newborns” (a common problem with excavated ancient populations) no correction was applied.

A systematic pathological examination was not performed on the skeletal material; only the observed traumatic lesions were described based on D. J. Ortner ${ }^{21}$ and V. L. Wedel and A. Galloway. ${ }^{22}$

The estimation of the biological distance between the late Roman period population of Somogyszil-Dögkúti dủló and other ancient populations was performed using the method elaborated by Penrose, ${ }^{23}$ based on the means of ten selected measurements (M1, M8, M9, M17, M45, M48, M51, M52, M54, M55) of the male skulls. For scale adjustment, the raw data were transformed with the standardised mean deviations of A. Thoma. ${ }^{24}$ According to the recommendation of I. Schwidetzky, ${ }^{25}$ only those series should be drawn into the PenRose biodistance calculation where the mean of every selected measurement is made up of at least seven data. We followed this recommendation in the case of the comparative materials, thus from the territory of the Carpathian Basin and from between the second to eighth centuries only those series were selected that conform to this criterion. This resulted in a total of 55 series for comparison. However, the Somogyszil-Dögkúti düló male cranial material itself fails to satisfy the above-mentioned criterion, as one of the selected cranial measurements (M17) is made up of only six individuals. Nonetheless, we chose to perform the biodistance calculations in order to determine how the Somogyszil-Dögkút population fits into the "framework" of other populations representing the territory and time interval chosen for the analysis. (Naturally, using an insufficient sample size may weaken the conclusions drawn from the analysis.) The relations of the Somogyszil-Dögkúti dủlő (male) population and its close analogies (below the 1\% and $2 \%$ error bands) were visualized with the help of a dendrogram that was created with the UPGMA (Unweighted Pair Group Method with Arithmetic Mean) hierarchical clustering method. ${ }^{26}$

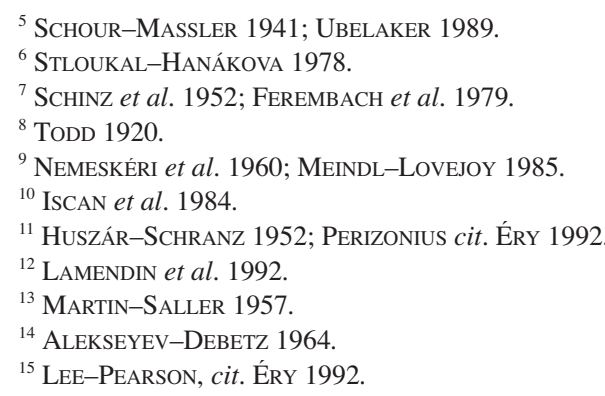

Acta Archaeologica Academiae Scientiarum Hungaricae 70, 2019

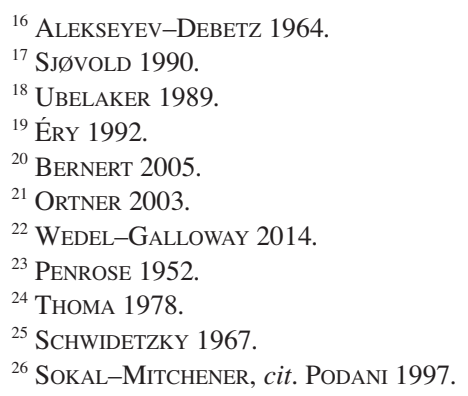

${ }^{16}$ Alerseyev-DeBetz 1964.

${ }^{19}$ ÉRY 1992.

${ }^{20}$ BERNERT 2005.

${ }^{22}$ Wedel-Galloway 2014

${ }^{23}$ PenRose 1952

${ }^{25}$ SCHWIDETZKY 1967

${ }^{26}$ SOKAL-Mitchener, cit. PoDANI 1997. 
RESULTS

\section{Demographic results}

The remains of 140 individuals were brought to light. The basic summary data of their sex and age distribution are presented in Table 1. The male/female sex ratio is more or less balanced (49 males, 56 females and 3 indeterminate individuals). The proportion of children as compared to adults is low, which can most likely be explained by the poor preservation of the anthropological material (Table 2). The mortality peak falls at the beginning of the maturus age in the case of males (40-44 years) and in the middle of the adultus age in the case of females

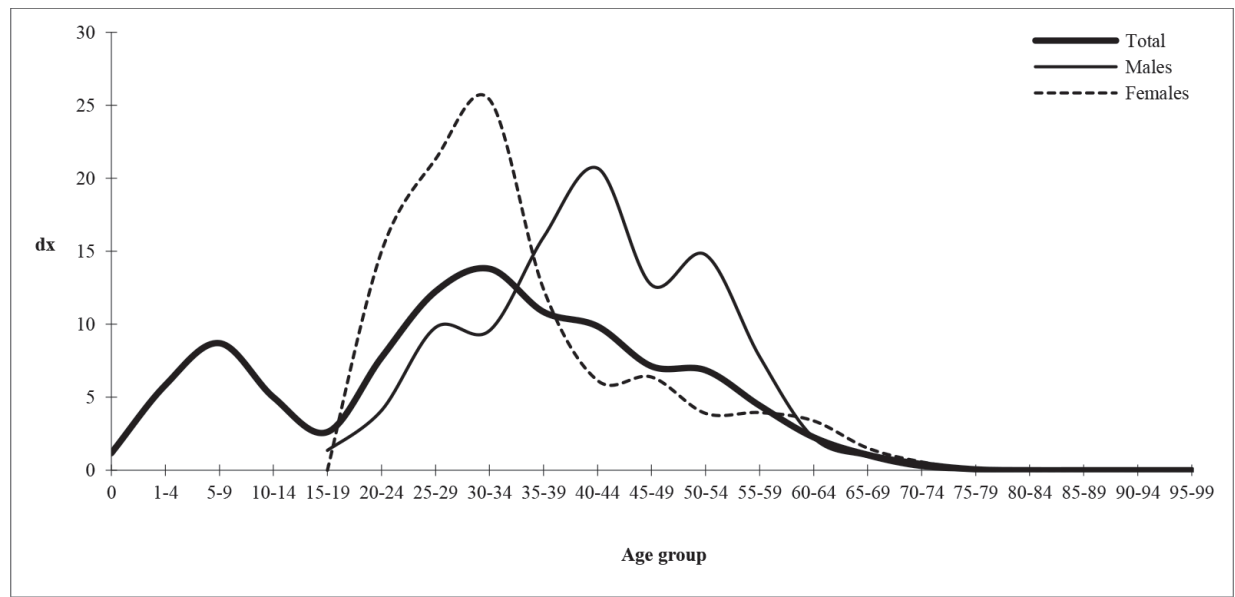

Fig. 1. The mortality curve of the Somogyszil-Dögkúti dűlő population

Table 1.

Sex and age distribution of the Somogyszil-Dögkúti dülő population

\begin{tabular}{|l|l|l|l|l|}
\hline Sex \Age group & Males & Females & Unknown & Total \\
\hline $\mathbf{1 - 6}$ & & & 12 & 12 \\
\hline $\mathbf{7 - 1 4}$ & & & 17 & 17 \\
\hline $\mathbf{1 5}-\mathbf{1 9}$ & 1 & & 3 & 1 \\
\hline $\mathbf{2 0 - 3 9}$ & 20 & 40 & & 4 \\
\hline $\mathbf{2 0 - 5 9}$ & 1 & 4 & & 61 \\
\hline $\mathbf{4 0 - 5 9}$ & 26 & 8 & & 5 \\
\hline $\mathbf{6 0 -}$ & 1 & 2 & 2 & 34 \\
\hline Unknown & & 2 & 35 & 3 \\
\hline Total & 49 & 56 & 4 \\
\hline
\end{tabular}

(30-34 year; Figure 1 and Table 3). This sexual difference in the mortality peak is generally observed among historical populations, and it is most likely an effect of the risks of childbearing (the possible complications of pregnancy and giving birth).

\section{Metric and morphological characteristics of the skulls}

In terms of morphological attributes (Table 4), the following traits are quite common in the population: the skull is ovoid in superior view, the occipital is curved, and the spina nasalis anterior is small or moderately developed. Rounded and rectangular-shaped orbits are equally frequent, and the morphology of the canine fossa is also 
Table 2.

Some basic data of the human skeletal material of Somogyszil-Dögkút

\begin{tabular}{|c|c|c|c|c|c|c|c|c|}
\hline Inventory number & Grave & Sex & Ag & & & Skull & Mandible & Postcranial skeleton \\
\hline 68.126.1. & 1 & female & 20 & - & 25 & incomplete & missing & incomplete \\
\hline 68.126 .2 & 2 & female & 60 & - & 65 & incomplete & incomplete & missing \\
\hline 68.126 .3 & 3 & male & 50 & - & 60 & incomplete & incomplete & incomplete \\
\hline 68.126 .4 & 4 & $?$ & 3 & - & 5 & incomplete & incomplete & incomplete \\
\hline 68.126 .5 & $5 a$ & male & 30 & - & 40 & well preserved & well preserved & incomplete \\
\hline 68.126 .6 & $5 b$ & male & 40 & - & 50 & incomplete & well preserved & incomplete \\
\hline 68.126 .7$. & 6 & $?$ & 7 & - & 8 & incomplete & missing & incomplete \\
\hline 68.126 .8$. & 7 & male & 50 & - & 60 & incomplete & incomplete & incomplete \\
\hline 68.126 .9$. & 8 & female & 40 & - & 75 & incomplete & incomplete & missing \\
\hline 68.126 .10 & 12 & male & 20 & - & 40 & missing & missing & incomplete \\
\hline 68.126 .11 & 13 & $?$ & 8 & - & 10 & missing & missing & incomplete \\
\hline 68.126 .12 & 14 & male & 20 & - & 60 & incomplete & incomplete & missing \\
\hline 68.126 .13 & 15 & male & 30 & - & 40 & incomplete & incomplete & incomplete \\
\hline 68.126 .14 & 16 & female & 25 & - & 35 & incomplete & incomplete & incomplete \\
\hline 68.126 .15 & 17 & male & 40 & - & 55 & incomplete & well preserved & well preserved \\
\hline 68.126 .16 & 18 & male & 25 & - & 30 & incomplete & incomplete & incomplete \\
\hline 68.126 .17$. & 19 & female & 60 & - & 70 & incomplete & incomplete & incomplete \\
\hline 68.126 .18$. & 20 & female & 25 & - & 35 & incomplete & well preserved & incomplete \\
\hline 68.126 .19$. & 21 & female & 30 & - & 40 & incomplete & well preserved & incomplete \\
\hline 68.126 .20$. & 23 & male & 30 & - & 40 & incomplete & incomplete & incomplete \\
\hline 68.126 .21$. & 24 & female & 30 & - & 35 & incomplete & incomplete & incomplete \\
\hline 68.126 .22 & 25 & $?$ & 15 & - & 18 & incomplete & incomplete & incomplete \\
\hline 68.126 .23 & 26 & female & 20 & - & 23 & incomplete & incomplete & incomplete \\
\hline 68.126 .24$. & 27 & male & 25 & - & 30 & missing & missing & well preserved \\
\hline 68.126 .25 & 29 & male & 40 & - & 45 & incomplete & incomplete & incomplete \\
\hline 68.126 .26 & 30 & $?$ & 15 & - & 18 & incomplete & incomplete & incomplete \\
\hline 68.126 .27$. & 31 & female & 25 & - & 30 & incomplete & incomplete & incomplete \\
\hline 68.126 .28 & 32 & female & 20 & - & 40 & incomplete & missing & missing \\
\hline 68.126 .29$. & 33 & male & 50 & - & 55 & incomplete & well preserved & incomplete \\
\hline 68.126 .30$. & 34 & $?$ & 9 & - & 11 & incomplete & well preserved & incomplete \\
\hline 68.126 .31$. & 35 & female & 55 & - & 60 & incomplete & incomplete & incomplete \\
\hline 68.126 .32$. & 36 & female & 25 & - & 35 & incomplete & incomplete & incomplete \\
\hline 68.126 .33 & 37 & female & 20 & - & 25 & incomplete & incomplete & incomplete \\
\hline 68.126 .34$. & 38 & female & 35 & - & 40 & well preserved & well preserved & well preserved \\
\hline 68.126 .35 & 39 & male & 40 & - & 45 & incomplete & well preserved & incomplete \\
\hline 68.126 .36$. & 40 & female & 20 & - & 25 & incomplete & well preserved & incomplete \\
\hline 68.126 .37$. & 41 & male & 50 & - & 60 & incomplete & well preserved & incomplete \\
\hline 68.126 .38 & 42 & $?$ & 6 & - & 7 & incomplete & missing & incomplete \\
\hline 68.126 .39$. & 43 & female & 50 & - & 60 & incomplete & incomplete & incomplete \\
\hline 68.126 .40$. & 44 & female & 35 & - & 45 & incomplete & incomplete & incomplete \\
\hline 68.126 .41$. & 45 & male & 35 & - & 40 & incomplete & incomplete & incomplete \\
\hline 68.126 .42$. & 46 & male & 40 & - & 50 & incomplete & incomplete & incomplete \\
\hline 68.126 .43$. & 48 & male & 40 & - & 45 & incomplete & well preserved & incomplete \\
\hline 68.126 .44$. & 49 & male & 60 & - & 70 & incomplete & incomplete & incomplete \\
\hline 68.126 .45$. & 50 & male & 50 & - & 55 & incomplete & incomplete & incomplete \\
\hline 68.126 .46$. & 51 & male & 35 & - & 40 & incomplete & incomplete & incomplete \\
\hline 68.126 .47$. & 52 & female & 25 & - & 35 & incomplete & incomplete & incomplete \\
\hline
\end{tabular}


Table 2.

Some basic data of the human skeletal material of Somogyszil-Dögkút (cont'd)

\begin{tabular}{|c|c|c|c|c|c|c|c|c|}
\hline Inventory number & Grave & Sex & Ag & & & Skull & Mandible & Postcranial skeleton \\
\hline 68.126 .48$. & 53 & male & 35 & - & 40 & well preserved & incomplete & incomplete \\
\hline 68.126 .49$. & 54 & $?$ & 7 & - & 9 & incomplete & incomplete & incomplete \\
\hline 68.126 .50$. & 55 & female & 25 & - & 35 & incomplete & incomplete & incomplete \\
\hline 68.126 .51$. & 56 & female & 20 & - & 40 & incomplete & missing & incomplete \\
\hline 68.126 .52 & 57 & male & 40 & - & 50 & incomplete & incomplete & incomplete \\
\hline 68.126 .53 & 58 & male & 20 & - & 40 & missing & missing & incomplete \\
\hline 68.126 .54 & 59 & male & 40 & - & 50 & incomplete & incomplete & incomplete \\
\hline 68.126 .55$. & 60 & male & 50 & - & 55 & incomplete & incomplete & incomplete \\
\hline 68.126 .56$. & 61 & female & 30 & - & 35 & well preserved & well preserved & incomplete \\
\hline 68.126 .57$. & 62 & female & 25 & - & 35 & incomplete & \begin{tabular}{|l|} 
incomplete \\
\end{tabular} & incomplete \\
\hline 68.126 .58$. & 63 & $?$ & 7 & - & 8 & incomplete & incomplete & incomplete \\
\hline 68.126 .59 & $64-65$ & $?$ & 4 & - & 5 & incomplete & incomplete & incomplete \\
\hline 68.126 .60$. & 66 & $?$ & 0 & - & 1 & incomplete & \begin{tabular}{|l|} 
incomplete \\
\end{tabular} & incomplete \\
\hline 68.126 .61$. & 67 & female & 25 & - & 30 & incomplete & incomplete & incomplete \\
\hline 68.126 .62 & 68 & female & 30 & - & 35 & incomplete & incomplete & incomplete \\
\hline 68.126 .63$. & 69 & $?$ & 9 & - & 10 & incomplete & incomplete & incomplete \\
\hline 68.126 .64$. & 70 & female & 30 & - & 40 & incomplete & incomplete & incomplete \\
\hline 68.126 .65$. & 71 & female & 45 & - & 55 & incomplete & incomplete & incomplete \\
\hline 68.126 .66$. & 72 & male & 50 & - & 60 & incomplete & incomplete & incomplete \\
\hline 68.126.67. & 73 & $?$ & 10 & - & 14 & missing & missing & incomplete \\
\hline 68.126 .68$. & 75 & female & 20 & - & 25 & incomplete & \begin{tabular}{|l|} 
incomplete \\
\end{tabular} & incomplete \\
\hline 68.126 .69$. & $75 a$ & male & 45 & - & 60 & incomplete & missing & incomplete \\
\hline 68.126 .70 . & 76 & $?$ & 20 & - & 40 & incomplete & missing & incomplete \\
\hline 68.126 .71$. & 77 & female & 25 & - & 30 & incomplete & incomplete & incomplete \\
\hline 68.126 .72$. & 78 & female & 20 & - & 75 & missing & missing & incomplete \\
\hline 68.126 .73 & 79 & male & 50 & - & 60 & incomplete & incomplete & incomplete \\
\hline 68.126 .74$. & 80 & female & 25 & - & 30 & incomplete & \begin{tabular}{|l|} 
incomplete \\
\end{tabular} & incomplete \\
\hline 68.126 .75 & 81 & $?$ & 15 & - & 18 & incomplete & incomplete & incomplete \\
\hline 68.126 .76$. & 82 & female & 30 & - & 40 & incomplete & incomplete & incomplete \\
\hline 68.126 .77$. & 84 & male & 30 & - & 40 & incomplete & \begin{tabular}{|l|} 
incomplete \\
\end{tabular} & incomplete \\
\hline 68.126 .78 & 86 & male & 20 & - & 25 & well preserved & well preserved & incomplete \\
\hline 68.126 .79 & 87 & $?$ & 7 & - & 9 & incomplete & incomplete & incomplete \\
\hline 68.126 .80$. & 88 & male & 20 & - & 40 & incomplete & incomplete & incomplete \\
\hline 68.126 .81$. & 89 & female & 25 & - & 30 & incomplete & \begin{tabular}{|l|} 
incomplete \\
\end{tabular} & incomplete \\
\hline 68.126 .82 & 90 & $?$ & 0 & - & 1 & incomplete & missing & missing \\
\hline 68.126 .83$. & 91 & $?$ & 3 & - & 4 & incomplete & missing & incomplete \\
\hline 68.126 .84$. & 92 & female & 20 & - & 25 & incomplete & incomplete & incomplete \\
\hline 68.126 .85$. & 93 & female & 30 & - & 35 & incomplete & \begin{tabular}{|l|} 
incomplete \\
\end{tabular} & incomplete \\
\hline 68.126 .86 & 94 & male & 30 & - & 40 & incomplete & missing & incomplete \\
\hline 68.126 .87$. & 95 & female & 20 & - & 30 & incomplete & incomplete & incomplete \\
\hline 68.126 .88$. & 96 & $?$ & 6 & - & 7 & incomplete & \begin{tabular}{|l|} 
incomplete \\
\end{tabular} & incomplete \\
\hline 68.126 .89$. & 97 & female & 40 & - & 60 & incomplete & incomplete & incomplete \\
\hline 68.126 .90 . & 98 & $?$ & 20 & - & 75 & missing & missing & incomplete \\
\hline 68.126 .91$. & 99 & $?$ & 2 & - & 6 & incomplete & incomplete & incomplete \\
\hline 68.126 .92$. & 100 & female & 20 & - & 60 & incomplete & incomplete & incomplete \\
\hline 68.126 .93 & 101 & $?$ & 3 & - & 4 & incomplete & incomplete & incomplete \\
\hline 68.126 .94$. & 102 & $?$ & 7 & - & 8 & incomplete & incomplete & incomplete \\
\hline 68.126 .95 . & 103 & male & 45 & - & 50 & incomplete & \begin{tabular}{|l|} 
incomplete \\
\end{tabular} & incomplete \\
\hline 68.126 .96 & 104 & male & 40 & - & 50 & incomplete & incomplete & incomplete \\
\hline 68.126 .97$. & 107 & $?$ & 12 & - & 14 & incomplete & incomplete & incomplete \\
\hline 68.126 .98$. & 108 & female & 30 & - & 35 & incomplete & well preserved & incomplete \\
\hline 68.126 .99 & 109 & female & 25 & - & 35 & well preserved & incomplete & incomplete \\
\hline
\end{tabular}


Table 2.

Some basic data of the human skeletal material of Somogyszil-Dögkút (cont'd)

\begin{tabular}{|c|c|c|c|c|c|c|c|c|}
\hline Inventory number & Grave & Sex & Ag & & & Skull & Mandible & Postcranial skeleton \\
\hline 68.126 .100$. & 110 & $?$ & 9 & - & 10 & incomplete & incomplete & incomplete \\
\hline 68.126 .101$. & 112 & male & 18 & - & 20 & well preserved & well preserved & incomplete \\
\hline 68.126 .102$. & 113/a & male & 40 & - & 50 & incomplete & incomplete & incomplete \\
\hline 68.126 .103$. & $113 / \mathrm{b}$ & male & 25 & - & 30 & well preserved & well preserved & missing \\
\hline 68.126 .104$. & 114 & male & 40 & - & 45 & incomplete & incomplete & incomplete \\
\hline 68.126 .105$. & 115 & female & 30 & - & 35 & incomplete & incomplete & incomplete \\
\hline 68.126 .106$. & 116 & male & 45 & - & 55 & incomplete & incomplete & incomplete \\
\hline 68.126 .107$. & 117 & male & 40 & - & 45 & incomplete & incomplete & incomplete \\
\hline 68.126 .108$. & 118 & female & 25 & - & 35 & incomplete & incomplete & incomplete \\
\hline 68.126 .109$. & 120 & female & 20 & - & 60 & missing & missing & incomplete \\
\hline 68.126 .110$. & 121 & female & 20 & - & 60 & missing & missing & incomplete \\
\hline 68.126 .111$. & 122 & $?$ & 0 & - & 1 & missing & missing & incomplete \\
\hline 68.126 .112 & 123 & female & 40 & - & 50 & incomplete & incomplete & incomplete \\
\hline 68.126 .113$. & 124 & male & 35 & - & 40 & incomplete & incomplete & incomplete \\
\hline 68.126 .114$. & 125 & $?$ & 11 & - & 13 & incomplete & incomplete & incomplete \\
\hline 68.126 .115$. & 126 & $?$ & 0 & - & 6 & incomplete & missing & incomplete \\
\hline 68.126 .116$. & 127 & female & 35 & - & 40 & incomplete & incomplete & incomplete \\
\hline 68.126 .117$. & 129 & female & 25 & - & 35 & incomplete & incomplete & incomplete \\
\hline 68.126 .118$. & 130 & male & 30 & - & 40 & well preserved & well preserved & incomplete \\
\hline 68.126 .119$. & 131 & female & 25 & - & 35 & incomplete & incomplete & incomplete \\
\hline 68.126 .120$. & sporadic & female & 45 & - & 50 & well preserved & well preserved & incomplete \\
\hline 68.126 .121$. & sporadic & male & 40 & - & 45 & incomplete & incomplete & missing \\
\hline 68.126 .122$. & sporadic & $?$ & 11 & - & 13 & incomplete & incomplete & incomplete \\
\hline 68.126 .123$. & sporadic & $?$ & 20 & - & 75 & missing & missing & incomplete \\
\hline 68.150 .1 & 132 & female & 40 & - & 50 & incomplete & incomplete & incomplete \\
\hline 68.150 .2$. & 133 & female & 30 & - & 35 & incomplete & incomplete & incomplete \\
\hline 68.150 .3 & 134 & $?$ & 12 & - & 14 & well preserved & well preserved & incomplete \\
\hline 68.150 .4$. & 136 & $?$ & 1 & - & 2 & incomplete & missing & incomplete \\
\hline 68.150 .5 . & 137 & female & 20 & - & 25 & well preserved & incomplete & incomplete \\
\hline 68.150 .6 . & 138 & male & 50 & - & 60 & well preserved & incomplete & incomplete \\
\hline 68.150 .7 & 139 & $?$ & 4 & - & 6 & incomplete & incomplete & incomplete \\
\hline 68.150 .8$. & 140 & male & 35 & - & 40 & incomplete & missing & incomplete \\
\hline 68.150 .9$. & 141 & female & 40 & - & 50 & incomplete & incomplete & incomplete \\
\hline 68.150 .10$. & $142 \mathrm{a}$ & $?$ & 6 & - & 7 & incomplete & missing & incomplete \\
\hline 68.150 .11$. & $142 b$ & $?$ & 1 & - & 2 & incomplete & missing & incomplete \\
\hline 68.150 .12$. & 143 & female & 30 & - & 40 & incomplete & incomplete & incomplete \\
\hline 68.126 .13$. & 144 & male & 40 & - & 50 & incomplete & well preserved & incomplete \\
\hline 68.150 .14 & 145 & female & 25 & - & 30 & incomplete & well preserved & incomplete \\
\hline 68.150 .15$. & 146 & male & 25 & - & 30 & incomplete & well preserved & well preserved \\
\hline 68.150 .16 & 147 & male & 25 & - & 35 & incomplete & well preserved & incomplete \\
\hline 68.150 .17$. & 148 & female & 20 & - & 22 & well preserved & well preserved & well preserved \\
\hline
\end{tabular}


Table 3.

Mortality table of the Somogyszil-Dögkút population

\begin{tabular}{|c|c|c|c|c|}
\hline \multirow[t]{2}{*}{ Age } & \multicolumn{2}{|l|}{ Dead } & \multirow{2}{*}{$\begin{array}{l}\text { Survivals } \\
\text { entering } \\
\text { (lx) } \\
\end{array}$} & \multirow{2}{*}{$\begin{array}{l}\text { Life expectancy } \\
(\mathrm{ex})\end{array}$} \\
\hline & Number (Dx) & Percentage (dx) & & \\
\hline \multicolumn{5}{|c|}{ Whole population } \\
\hline 0 & 1.6 & 1.17 & 100.00 & 31.77 \\
\hline $1-4$ & 8.2 & 5.84 & 98.83 & 31.14 \\
\hline $5-9$ & 12.2 & 8.70 & 92.99 & 28.97 \\
\hline $10-14$ & 7.0 & 5.00 & 84.29 & 26.70 \\
\hline $15-19$ & 3.7 & 2.62 & 79.29 & 23.22 \\
\hline $20-24$ & 10.8 & 7.72 & 76.67 & 18.93 \\
\hline $25-29$ & 17.1 & 12.24 & 68.95 & 15.77 \\
\hline $30-34$ & 19.3 & 13.80 & 56.71 & 13.64 \\
\hline $35-39$ & 15.2 & 10.85 & 42.91 & 12.22 \\
\hline $40-44$ & 13.8 & 9.86 & 32.06 & 10.51 \\
\hline $45-49$ & 10.0 & 7.12 & 22.20 & 9.06 \\
\hline $50-54$ & 9.6 & 6.85 & 15.08 & 7.16 \\
\hline $55-59$ & 6.2 & 4.43 & 8.23 & 6.05 \\
\hline $60-64$ & 3.2 & 2.26 & 3.79 & 5.19 \\
\hline $65-69$ & 1.5 & 1.06 & 1.54 & 4.15 \\
\hline $70-74$ & 0.6 & 0.42 & 0.48 & 2.80 \\
\hline $75-79$ & 0.1 & 0.06 & 0.06 & 2.50 \\
\hline Total & 140.0 & 100.00 & & \\
\hline \multicolumn{5}{|l|}{ Males } \\
\hline $15-19$ & 0.7 & 1.36 & 100 & 27.13 \\
\hline $20-24$ & 2.0 & 4.09 & 98.64 & 22.47 \\
\hline $25-29$ & 4.8 & 9.78 & 94.55 & 18.33 \\
\hline $30-34$ & 4.7 & 9.56 & 84.77 & 15.16 \\
\hline $35-39$ & 7.8 & 15.96 & 75.21 & 11.77 \\
\hline $40-44$ & 10.1 & 20.69 & 59.25 & 9.27 \\
\hline $45-49$ & 6.2 & 12.69 & 38.56 & 7.90 \\
\hline $50-54$ & 7.2 & 14.76 & 25.88 & 5.54 \\
\hline $55-59$ & 3.8 & 7.79 & 11.12 & 4.58 \\
\hline $60-64$ & 1.1 & 2.22 & 3.33 & 4.45 \\
\hline $65-69$ & 0.5 & 0.93 & 1.11 & 3.33 \\
\hline $70-74$ & 0.1 & 0.19 & 0.19 & 2.50 \\
\hline $75-79$ & 0.0 & 0.00 & 0.00 & 0.00 \\
\hline Total & 49.0 & 100.00 & & \\
\hline \multicolumn{5}{|c|}{ Females } \\
\hline $15-19$ & 0.0 & 0.00 & 100 & 20.71 \\
\hline $20-24$ & 8.4 & 14.97 & 100.00 & 15.71 \\
\hline $25-29$ & 11.9 & 21.31 & 85.03 & 13.03 \\
\hline $30-34$ & 14.2 & 25.39 & 63.72 & 11.55 \\
\hline $35-39$ & 6.9 & 12.40 & 38.33 & 12.55 \\
\hline $40-44$ & 3.4 & 6.15 & 25.92 & 12.36 \\
\hline $45-49$ & 3.6 & 6.38 & 19.78 & 10.42 \\
\hline $50-54$ & 2.2 & 3.89 & 13.39 & 9.20 \\
\hline $55-59$ & 2.2 & 3.95 & 9.50 & 6.95 \\
\hline
\end{tabular}


Table 3.

Mortality table of the Somogyszil-Dögkút population (cont'd)

\begin{tabular}{|l|l|l|l|l|}
\hline \multirow{2}{*}{ Age } & \multicolumn{2}{|l|}{ Dead } & $\begin{array}{l}\text { Survivals } \\
\text { entering } \\
(\mathbf{l x})\end{array}$ & $\begin{array}{l}\text { Life expectancy } \\
(\mathbf{e x})\end{array}$ \\
\cline { 2 - 5 } & Number $(\mathbf{D x})$ & Percentage $(\mathbf{d x})$ & 5.55 & 5.11 \\
\hline $60-64$ & 1.9 & 3.38 & 2.17 & 4.19 \\
\hline $65-69$ & 0.8 & 1.52 & 0.65 & 3.13 \\
\hline $70-74$ & 0.3 & 0.57 & 0.08 & 2.50 \\
\hline $75-79$ & 0.0 & 0.08 & & \\
\hline Total & 56.0 & 100.00 & & \\
\hline
\end{tabular}

varied. The presence of maxillary torus is quite rare, whereas no shovel-shaped incisors occurs in the studied material. There are differences in the frequencies of palatinus torus and fossa praenasalis as well as in the occurrence of the curved and straight foreheads between the two sexes.

The metric data and indices of the skulls are presented in Tables 5-6, while Table 7 shows the summary statistics of the skulls.

On the basis of the cranial indices, the majority of the late Roman community of Somogyszil-Dögkút can be characterized by a medium long/long (length-width index), low/moderately high (length-height index), and moderately high/high (height-width index) skull. Male foreheads are most often moderately wide or wide (transversal-frontoparietal index), whereas this index shows greater variability among females. Cranial capacity falls into the moderately large/large categories in both sexes. The face and upper face are medium wide/medium high in around half of the cases. Male orbits are usually low, whereas female orbits are low/moderately high. Nasal width varies in both sexes (Table 8).

For the calculation of the mean sigma ratio only the sigma ratios of those cranial measurements and indices were used that were composed of at least seven individual data. The mean sigma ratio of the cranial measurements is 103.53 for male skulls, and 102.08 for female skulls. The value for the cranial indices is 112.18 for males, and 137.21 for females respectively. Thus, the variability of the linear measurements (bearing information mainly about cranial size) indicates a natural population (the theoretical value for an average level of heterogeneity is 100.00), however the sigma ratios of the indices (bearing information about cranial shape) show a more mixed population, particularly for females, with a greater level of heterogeneity than that is typical for a natural population. This may suggest that the community was composed not just by local people, but some extralocal gene flow (immigrants that differed from the locals in their cranial shapes) were also present in it.

It is important to point out that due to the combined effect of the relatively small sample size and the poor preservation of the skeletal material the number of recorded cranial measurements and morphological attributes are rather small. Thus, every above mentioned result, and the conclusions drawn from it should be handled with caution as the small sample size goes along with an increased possibility of random effects that may influence the results.

Based on morphoscopic traits, every examinable skull belongs to the Caucasoid group. With respect to the traditional (and quite subjective, thus, in our opinion, "semi-scientific") racial typology, it can be stated that Nordoid/Mediterranoid, Cromagnoid and archaic chamaecran types occurred in the population (Figs 2-6).

Mention must be made of the characteristics of the skulls recovered from burials whose S-N/N-S orientation differed from the period's norm. Due to their poor preservation (which was among the worst in the excavated skeletal material) only a few measurements of a single female skull could be taken (Grave no. 70, Fig. 7), although the form and major proportions of the braincase of two further male skulls (Graves no. 41 and no. 50) could also be estimated, despite their fragmentation. A shared trait of these three skulls is that the length/width index of every one of them falls into the short (brachycran or hyperbrachycran) class category. The frequency of brachycran skulls is rather low in the cemetery: disregarding the female interred in Grave no. 70, from the altogether 32 skulls suitable to calculate the length/width index, only four fall into the brachycran/hyperbrachycran category. This marked difference suggests that these $\mathrm{S}-\mathrm{N} / \mathrm{N}-\mathrm{S}$ oriented graves contained the burials of a (smaller) immigrant group whose anthropological characteristics differed in certain aspects from the local population. This possibility is indirectly further strengthened with the stature data presented below. In the lack of measurable/examinable skulls, we cannot use any traditional anthropological approach to identify the origin of this possibly immigrant group, but - to make 
Table 4.

Distribution of the morphological characteristics of the skull in the population of Somogyszil-Dögkút

\begin{tabular}{|c|c|c|c|c|c|c|c|}
\hline \multicolumn{2}{|l|}{ Characteristics } & \multicolumn{2}{|c|}{ Males } & \multicolumn{2}{|c|}{ Females } & \multicolumn{2}{|c|}{ Together } \\
\hline & & $\mathbf{N}$ & $\%$ & $\mathbf{N}$ & $\%$ & $\mathbf{N}$ & $\%$ \\
\hline $\begin{array}{l}\text { Cranium } \\
\text { (norma verticalis) }\end{array}$ & $\begin{array}{l}\text { Ellipsoid } \\
\text { Ovoid } \\
\text { Pentagonoid } \\
\text { Sphenoid } \\
\text { Spheroid } \\
\text { Rhomboid }\end{array}$ & $\begin{array}{r}4 \\
15 \\
3 \\
0 \\
0 \\
2 \\
\end{array}$ & $\begin{array}{r}16.67 \\
62.50 \\
12.50 \\
0.00 \\
0.00 \\
8.33 \\
\end{array}$ & $\begin{array}{r}3 \\
21 \\
1 \\
0 \\
0 \\
0\end{array}$ & $\begin{array}{r}12.00 \\
84.00 \\
4.00 \\
0.00 \\
0.00 \\
0.00 \\
\end{array}$ & $\begin{array}{r}7 \\
36 \\
4 \\
0 \\
0 \\
2 \\
\end{array}$ & $\begin{array}{r}14.29 \\
73.47 \\
8.16 \\
0.00 \\
0.00 \\
4.08 \\
\end{array}$ \\
\hline $\boldsymbol{\Sigma}$ & & 24 & & 25 & & 49 & \\
\hline Forehead & $\begin{array}{l}\text { Straight } \\
\text { Curved } \\
\text { Sloped } \\
\end{array}$ & $\begin{array}{r}5 \\
24 \\
1 \\
\end{array}$ & $\begin{array}{r}16.67 \\
80.00 \\
3.33 \\
\end{array}$ & $\begin{array}{r}18 \\
10 \\
1 \\
\end{array}$ & $\begin{array}{r}62.07 \\
34.48 \\
3.45 \\
\end{array}$ & $\begin{array}{r}23 \\
34 \\
2 \\
\end{array}$ & $\begin{array}{r}38.98 \\
57.63 \\
3.39 \\
\end{array}$ \\
\hline $\mathbf{\Sigma}$ & & 30 & & 29 & & 59 & \\
\hline Occipital & $\begin{array}{l}\text { Bathrocran } \\
\text { Curvoccipital } \\
\text { Planoccipital } \\
\end{array}$ & $\begin{array}{r}4 \\
23 \\
0 \\
\end{array}$ & $\begin{array}{r}14.81 \\
85.19 \\
0.00 \\
\end{array}$ & $\begin{array}{r}4 \\
20 \\
1 \\
\end{array}$ & $\begin{array}{r}16.00 \\
80.00 \\
4.00 \\
\end{array}$ & $\begin{array}{r}8 \\
43 \\
1 \\
\end{array}$ & $\begin{array}{r}15.39 \\
82.69 \\
1.92 \\
\end{array}$ \\
\hline $\mathbf{\Sigma}$ & & 27 & & 25 & & 52 & \\
\hline Orbits & $\begin{array}{l}\text { Rounded } \\
\text { Rectangular }\end{array}$ & $\begin{array}{l}6 \\
6 \\
\end{array}$ & $\begin{array}{l}50.00 \\
50.00 \\
\end{array}$ & $\begin{array}{r}11 \\
8 \\
\end{array}$ & $\begin{array}{l}57.89 \\
42.11 \\
\end{array}$ & $\begin{array}{l}17 \\
14 \\
\end{array}$ & $\begin{array}{l}54.84 \\
45.16 \\
\end{array}$ \\
\hline$\Sigma$ & & 12 & & 19 & & 31 & \\
\hline Lower margin of the apertura piriformis & $\begin{array}{l}\text { Anthropin } \\
\text { Fossa praenasalis } \\
\text { Sulcus praenasalis }\end{array}$ & $\begin{array}{r}10 \\
8 \\
0 \\
\end{array}$ & $\begin{array}{r}55.56 \\
44.44 \\
0.00 \\
\end{array}$ & $\begin{array}{r}13 \\
3 \\
0 \\
\end{array}$ & $\begin{array}{r}81.25 \\
18.75 \\
0.00 \\
\end{array}$ & $\begin{array}{r}23 \\
11 \\
0 \\
\end{array}$ & $\begin{array}{r}67.65 \\
32.35 \\
0.00 \\
\end{array}$ \\
\hline $\mathbf{\Sigma}$ & & 18 & & 16 & & 34 & \\
\hline Spina nasalis anterior & $\begin{array}{l}\text { Broca } 1 \\
\text { Broca } 2 \\
\text { Broca } 3 \\
\text { Broca } 4 \\
\text { Broca } 5 \\
\end{array}$ & $\begin{array}{l}0 \\
5 \\
2 \\
2 \\
1 \\
\end{array}$ & $\begin{array}{r}0.00 \\
50.00 \\
20.00 \\
20.00 \\
10.00 \\
\end{array}$ & $\begin{array}{l}0 \\
6 \\
3 \\
0 \\
0\end{array}$ & $\begin{array}{r}0.00 \\
66.67 \\
33.33 \\
0.00 \\
0.00 \\
\end{array}$ & $\begin{array}{r}0 \\
11 \\
5 \\
2 \\
1 \\
\end{array}$ & $\begin{array}{r}0.00 \\
57.89 \\
26.32 \\
10.53 \\
5.26 \\
\end{array}$ \\
\hline $\boldsymbol{\Sigma}$ & & 10 & & 9 & & 19 & \\
\hline Alveolar prognathism & $\begin{array}{l}\text { Not present } \\
\text { Moderate } \\
\text { Strong } \\
\end{array}$ & $\begin{array}{l}5 \\
8 \\
0 \\
\end{array}$ & $\begin{array}{r}38.46 \\
61.54 \\
0.00 \\
\end{array}$ & $\begin{array}{l}9 \\
4 \\
0 \\
\end{array}$ & $\begin{array}{r}69.23 \\
30.77 \\
0.00 \\
\end{array}$ & $\begin{array}{r}14 \\
12 \\
0 \\
\end{array}$ & $\begin{array}{r}53.85 \\
46.15 \\
0.00 \\
\end{array}$ \\
\hline $\mathbf{\Sigma}$ & & 13 & & 13 & & 26 & \\
\hline Torus palatinus & $\begin{array}{l}\text { Not present } \\
\text { Moderate } \\
\text { Strong } \\
\end{array}$ & $\begin{array}{l}4 \\
3 \\
0 \\
\end{array}$ & $\begin{array}{r}57.14 \\
42.86 \\
0.00 \\
\end{array}$ & $\begin{array}{l}4 \\
0 \\
0 \\
\end{array}$ & $\begin{array}{r}100.00 \\
0.00 \\
0.00 \\
\end{array}$ & $\begin{array}{l}8 \\
3 \\
0 \\
\end{array}$ & $\begin{array}{r}72.73 \\
27.27 \\
0.00 \\
\end{array}$ \\
\hline $\mathbf{\Sigma}$ & & 7 & & 4 & & 11 & \\
\hline Torus maxillaris & $\begin{array}{l}\text { Not present } \\
\text { Moderate } \\
\text { Strong } \\
\end{array}$ & $\begin{array}{r}15 \\
1 \\
0 \\
\end{array}$ & $\begin{array}{r}93.75 \\
6.25 \\
0.00 \\
\end{array}$ & $\begin{array}{r}10 \\
0 \\
0\end{array}$ & $\begin{array}{r}100.00 \\
0.00 \\
0.00 \\
\end{array}$ & $\begin{array}{r}25 \\
1 \\
0 \\
\end{array}$ & $\begin{array}{r}96.15 \\
3.85 \\
0.00 \\
\end{array}$ \\
\hline$\Sigma$ & & 16 & & 10 & & 26 & \\
\hline Shovel-shaped incisor & $\begin{array}{l}\text { Not present } \\
\text { Present }\end{array}$ & $\begin{array}{l}3 \\
0 \\
\end{array}$ & $\begin{array}{r}100.00 \\
0.00 \\
\end{array}$ & $\begin{array}{l}3 \\
0 \\
\end{array}$ & $\begin{array}{r}100.00 \\
0.00 \\
\end{array}$ & $\begin{array}{l}6 \\
0 \\
\end{array}$ & $\begin{array}{r}100.00 \\
0.00 \\
\end{array}$ \\
\hline $\boldsymbol{\Sigma}$ & & 3 & & 3 & & 6 & \\
\hline Canine fossa & $\begin{array}{l}\text { Shallow } \\
\text { Moderately deep } \\
\text { Deep }\end{array}$ & $\begin{array}{l}9 \\
5 \\
6 \\
\end{array}$ & $\begin{array}{l}45.00 \\
25.00 \\
30.00 \\
\end{array}$ & $\begin{array}{r}5 \\
11 \\
3 \\
\end{array}$ & $\begin{array}{l}26.32 \\
57.89 \\
15.79 \\
\end{array}$ & $\begin{array}{r}14 \\
16 \\
9 \\
\end{array}$ & $\begin{array}{l}35.90 \\
41.02 \\
23.08 \\
\end{array}$ \\
\hline $\mathbf{\Sigma}$ & & 20 & & 19 & & 39 & \\
\hline
\end{tabular}


Table 5.

Male cranial measurements and indices in the population of Somogyszil-Dögkút

\begin{tabular}{|c|c|c|c|c|c|c|c|c|c|c|c|c|}
\hline \multirow{2}{*}{$\begin{array}{l}\text { Martin } \\
\text { No. }\end{array}$} & \multicolumn{12}{|c|}{ Grave No. } \\
\hline & 3 & $5 / a$ & $5 / \mathrm{b}$ & 7 & 17 & 23 & 29 & 33 & 39 & 41 & 45 & 48 \\
\hline 1 & 180 & 197 & 183 & - & 188 & - & 170 & 183 & - & - & - & 181 \\
\hline 5 & - & 104 & 102 & - & - & - & - & 102 & - & - & - & - \\
\hline 8 & 138 & 136 & 141 & - & 133 & - & - & 137 & - & - & - & 134 \\
\hline 9 & - & 99 & 96 & - & 95 & - & 100 & 96 & - & - & - & - \\
\hline 10 & - & 121 & 120 & - & 116 & - & - & 115 & - & - & - & - \\
\hline 11 & - & 124 & 130 & - & 118 & - & - & 125 & - & - & - & - \\
\hline 12 & 103 & 110 & 112 & 111 & 108 & - & - & 112 & - & - & - & - \\
\hline 17 & - & 139 & 133 & - & - & - & - & 136 & - & - & - & - \\
\hline 20 & - & 116 & 108 & - & 110 & - & - & 113 & - & - & - & 114 \\
\hline 38 & - & 1494 & 1376 & - & 1363 & - & - & 1393 & - & - & - & 1369 \\
\hline 40 & - & 103 & - & - & - & - & - & - & - & - & - & - \\
\hline 43 & - & 107 & 106 & - & 105 & 106 & 105 & 103 & - & - & - & 109 \\
\hline 45 & - & 133 & 140 & - & - & - & - & 136 & - & - & - & 131 \\
\hline 46 & - & 100 & - & - & - & - & - & - & - & - & - & 97 \\
\hline 47 & - & 115 & - & - & - & - & - & - & - & - & - & 121 \\
\hline 48 & - & 72 & - & - & - & - & - & - & - & - & - & 71 \\
\hline 51 & - & 43 & 42 & - & - & - & - & 42 & 42 & - & - & 45 \\
\hline 52 & - & 33 & 35 & - & - & - & - & 30 & - & - & - & 30 \\
\hline 54 & - & - & - & - & - & - & - & - & 27 & - & - & 26 \\
\hline 55 & - & - & - & - & - & - & - & - & 48 & - & - & 51 \\
\hline 62 & - & 51 & - & - & - & - & - & - & 47 & - & - & 47 \\
\hline 63 & - & - & - & - & - & - & - & - & - & - & - & 42 \\
\hline 65 & - & 126 & - & - & 120 & - & - & 129 & 137 & - & 125 & - \\
\hline 66 & - & - & 111 & - & 102 & - & - & 115 & 105 & 98 & 105 & - \\
\hline 69 & - & 33 & - & - & 31 & - & - & 36 & 37 & 31 & 35 & 38 \\
\hline 70 & - & 68 & 69 & - & 58 & - & - & 62 & 70 & 62 & 62 & 62 \\
\hline 71 & - & 39 & 30 & 28 & 29 & - & 31 & 33 & 34 & 31 & 32 & 36 \\
\hline $8: 1$ & 76.67 & 69.04 & 77.05 & - & 70.74 & - & - & 74.86 & - & - & - & 74.03 \\
\hline $17: 1$ & - & 70.56 & 72.68 & - & - & - & - & 74.32 & - & - & - & - \\
\hline $17: 8$ & - & 102.21 & 94.33 & - & - & - & - & 99.27 & - & - & - & - \\
\hline $20: 1$ & - & 58.88 & 59.02 & - & 58.51 & - & - & 61.75 & - & - & - & 62.98 \\
\hline $20: 8$ & - & 85.29 & 76.60 & - & 82.71 & - & - & 82.48 & - & - & - & 85.07 \\
\hline $9: 8$ & - & 72.79 & 68.09 & - & 71.43 & - & - & 70.07 & - & - & - & - \\
\hline 47:45 & - & 86.47 & - & - & - & - & - & - & - & - & - & 92.37 \\
\hline 48:45 & - & 54.14 & - & - & - & - & - & - & - & - & - & 54.20 \\
\hline $52: 51$ & - & 76.74 & 83.33 & - & - & - & - & 71.43 & - & - & - & 66.67 \\
\hline 54:55 & - & - & - & - & - & - & - & - & 56.25 & - & - & 50.98 \\
\hline $63: 62$ & - & - & - & - & - & - & - & - & - & - & - & 89.36 \\
\hline
\end{tabular}


Table 5.

Male cranial measurements and indices in the population of Somogyszil-Dögkút (cont'd)

\begin{tabular}{|c|c|c|c|c|c|c|c|c|c|c|c|c|}
\hline \multirow{2}{*}{\begin{tabular}{|l|} 
Martin \\
No.
\end{tabular}} & \multicolumn{12}{|c|}{ Grave No. } \\
\hline & 49 & 50 & 51 & 53 & 57 & 59 & 60 & 79 & 84 & 86 & 103 & $113 / a$ \\
\hline 1 & 192 & 176 & 192 & 186 & - & - & - & 190 & 197 & 175 & - & 193 \\
\hline 5 & - & - & - & 101 & - & - & - & - & 106 & 96 & - & - \\
\hline 8 & 136 & - & - & 135 & - & - & - & 150 & 144 & 135 & - & 149 \\
\hline 9 & 91 & 98 & - & 89 & - & - & 101 & 101 & 103 & 90 & - & 101 \\
\hline 10 & - & 120 & - & 114 & - & - & 121 & - & 123 & 109 & - & 133 \\
\hline 11 & - & - & - & 115 & - & - & - & 135 & 127 & 118 & - & 123 \\
\hline 12 & - & - & - & 110 & - & - & - & 118 & 122 & 106 & - & 113 \\
\hline 17 & - & - & - & 128 & - & - & - & - & 140 & 135 & - & - \\
\hline 20 & - & - & - & 106 & - & - & - & 114 & 116 & 114 & - & - \\
\hline 38 & 192 & 176 & 192 & 186 & - & - & - & 190 & 197 & 175 & - & 193 \\
\hline 40 & - & - & - & 1331 & - & - & - & 1545 & 1560 & 1342 & - & - \\
\hline 43 & - & - & - & 92 & - & - & - & - & - & 91 & - & - \\
\hline 45 & 98 & 107 & - & 101 & - & - & 107 & 115 & 113 & 99 & - & 109 \\
\hline 46 & - & - & - & 126 & - & - & - & - & 134 & 127 & - & - \\
\hline 47 & - & - & - & 91 & - & - & - & - & - & 93 & - & - \\
\hline 48 & - & - & - & 114 & - & - & - & - & - & 116 & - & - \\
\hline 51 & - & - & - & 71 & - & - & - & - & - & 67 & - & - \\
\hline 52 & - & - & - & 40 & - & - & - & - & - & 41 & - & - \\
\hline 54 & - & - & - & 34 & - & - & - & - & - & 29 & - & - \\
\hline 55 & - & - & - & 25 & - & - & - & - & - & 23 & - & - \\
\hline 62 & - & - & - & 44 & - & - & - & - & - & - & - & - \\
\hline 63 & - & - & - & - & - & - & - & - & - & 43 & - & - \\
\hline 65 & - & - & - & - & - & - & - & - & - & 123 & - & - \\
\hline 66 & - & - & - & 109 & - & - & - & - & - & 100 & - & - \\
\hline 69 & - & - & 33 & 33 & - & - & 34 & - & - & 34 & 34 & 31 \\
\hline 70 & - & - & - & 67 & 66 & 71 & 61 & 73 & 63 & 58 & 62 & 58 \\
\hline 71 & - & - & 34 & 30 & 31 & 33 & 35 & 33 & 33 & 32 & 33 & 35 \\
\hline $8: 1$ & 70.83 & - & - & 72.58 & - & - & - & 78.95 & 73.10 & 77.14 & - & 77.20 \\
\hline $17: 1$ & - & - & - & 68.82 & - & - & - & - & 71.07 & 77.14 & - & - \\
\hline $17: 8$ & - & - & - & 94.81 & - & - & - & - & 97.22 & 100.00 & - & - \\
\hline $20: 1$ & - & - & - & 56.99 & - & - & - & 60.00 & 58.88 & 65.14 & - & - \\
\hline $20: 8$ & - & - & - & 78.52 & - & - & - & 76.00 & 80.56 & 84.44 & - & - \\
\hline $9: 8$ & 66.91 & - & - & 65.93 & - & - & - & 67.33 & 71.53 & 66.67 & - & 67.79 \\
\hline $47: 45$ & - & - & - & 90.48 & - & - & - & - & - & 91.34 & - & - \\
\hline $48: 45$ & - & - & - & 56.35 & - & - & - & - & - & 52.76 & - & - \\
\hline $52: 51$ & - & - & - & 85.00 & - & - & - & - & - & 70.73 & - & - \\
\hline $54: 55$ & - & - & - & 47.17 & - & - & - & - & - & 46.00 & - & - \\
\hline $63: 62$ & - & - & - & - & - & - & - & - & - & - & - & - \\
\hline
\end{tabular}


Table 5.

Male cranial measurements and indices in the population of Somogyszil-Dögkút (cont'd)

\begin{tabular}{|c|c|c|c|c|c|c|c|c|c|c|c|}
\hline \multirow{2}{*}{$\begin{array}{l}\text { Martin } \\
\text { No. }\end{array}$} & \multicolumn{11}{|c|}{ Grave No. } \\
\hline & $113 / \mathrm{b}$ & 114 & 116 & 117 & 124 & 130 & 138 & 140 & 144 & 146 & 147 \\
\hline 1 & 184 & - & - & - & 187 & 181 & 183 & - & - & 194 & 182 \\
\hline 5 & 95 & - & - & - & - & - & - & - & - & - & - \\
\hline 8 & 149 & - & - & - & 138 & 152 & 135 & - & 145 & 146 & 138 \\
\hline 9 & 97 & 99 & - & 100 & 97 & 89 & 96 & 93 & - & 105 & 94 \\
\hline 10 & 119 & - & - & - & 119 & - & 116 & 120 & - & 125 & 117 \\
\hline 11 & 130 & - & - & - & 123 & 126 & 123 & - & - & 128 & 122 \\
\hline 12 & 118 & - & - & - & 106 & 118 & 114 & - & 117 & - & 107 \\
\hline 17 & - & - & - & - & - & - & - & - & - & - & - \\
\hline 20 & 115 & - & - & - & - & 111 & 118 & - & - & 122 & - \\
\hline 38 & 1510 & - & - & - & - & 1474 & 1423 & - & - & 1621 & - \\
\hline 40 & - & - & - & - & - & - & - & - & - & - & - \\
\hline 43 & 108 & - & - & 108 & 107 & 98 & 105 & 103 & - & 111 & 102 \\
\hline 45 & 133 & - & - & - & - & - & 135 & - & - & 144 & - \\
\hline 46 & 97 & - & - & - & 95 & - & - & - & - & 103 & - \\
\hline 47 & 106 & - & - & - & 125 & 126 & - & - & - & 118 & - \\
\hline 48 & 66 & - & - & - & 79 & 74 & 63 & 69 & - & 74 & - \\
\hline 51 & 44 & - & - & - & 41 & 40 & 42 & 43 & - & 45 & - \\
\hline 52 & 34 & - & - & - & - & 31 & 30 & 36 & - & 33 & - \\
\hline 54 & 24 & - & - & - & - & 26 & 25 & 26 & - & 23 & - \\
\hline 55 & 48 & - & - & - & - & 53 & 44 & 56 & - & 52 & - \\
\hline 62 & 43 & - & - & - & - & - & 48 & - & - & 46 & - \\
\hline 63 & 45 & - & - & - & - & - & - & - & - & 43 & - \\
\hline 65 & 123 & - & - & - & 120 & 127 & - & - & - & 122 & - \\
\hline 66 & 108 & - & - & - & 107 & 105 & - & - & - & 103 & 105 \\
\hline 69 & 26 & 30 & - & - & 29 & 34 & 36 & - & - & 34 & 29 \\
\hline 70 & - & 55 & 74 & 55 & 52 & 66 & 64 & - & 66 & 70 & 60 \\
\hline 71 & 29 & 30 & 30 & 32 & 32 & 31 & 40 & - & 31 & 36 & 27 \\
\hline $8: 1$ & 80.98 & - & - & - & 73.80 & 83.98 & 73.77 & - & - & 75.26 & 75.82 \\
\hline $17: 1$ & - & - & - & - & - & - & - & - & - & - & - \\
\hline $17: 8$ & - & - & - & - & - & - & - & - & - & - & - \\
\hline $20: 1$ & 62.50 & - & - & - & - & 61.33 & 64.48 & - & - & 62.89 & - \\
\hline $20: 8$ & 77.18 & - & - & - & - & 73.03 & 87.41 & - & - & 83.56 & - \\
\hline $9: 8$ & 65.10 & - & - & - & 70.29 & 58.55 & 71.11 & - & - & 71.92 & 68.12 \\
\hline $47: 45$ & 79.70 & - & - & - & - & - & - & - & - & 81.94 & - \\
\hline 48:45 & 49.62 & - & - & - & - & - & 46.67 & - & - & 51.39 & - \\
\hline $52: 51$ & 77.27 & - & - & - & - & 77.50 & 71.43 & 83.72 & - & 73.33 & - \\
\hline $54: 55$ & 50.00 & - & - & - & - & 49.06 & 56.82 & 46.43 & - & 44.23 & - \\
\hline $63: 62$ & 104.65 & - & - & - & - & - & - & - & - & 93.48 & - \\
\hline
\end{tabular}


Table 6.

Female cranial measurements and indices in the population of Somogyszil-Dögkút

\begin{tabular}{|c|c|c|c|c|c|c|c|c|c|c|c|c|}
\hline \multirow{2}{*}{$\begin{array}{l}\text { Martin } \\
\text { No. }\end{array}$} & \multicolumn{12}{|c|}{ Grave No. } \\
\hline & 2 & 19 & 20 & 21 & 24 & 35 & 38 & 40 & 43 & 44 & 55 & 61 \\
\hline 1 & 186 & - & - & 174 & - & 181 & 191 & - & 185 & 186 & - & 182 \\
\hline 5 & - & - & - & - & - & - & 102 & - & - & - & - & 93 \\
\hline 8 & 125 & - & - & 141 & - & - & 138 & - & 141 & - & - & 143 \\
\hline 9 & 93 & 95 & 90 & 89 & 96 & 88 & 95 & 92 & 93 & 99 & 90 & 94 \\
\hline 10 & 112 & 123 & - & 117 & - & - & 116 & - & 117 & - & 117 & 123 \\
\hline 11 & - & - & - & 118 & - & - & 125 & - & - & - & - & 126 \\
\hline 12 & - & - & - & 107 & - & 107 & 116 & - & 110 & - & - & 108 \\
\hline 17 & - & - & - & - & - & - & 136 & - & - & - & - & 115 \\
\hline 20 & - & - & - & 104 & - & 108 & 112 & - & - & - & - & 100 \\
\hline 38 & - & - & - & 1253 & - & - & 1403 & - & - & - & - & 1272 \\
\hline 40 & - & - & - & - & - & - & 94 & - & - & - & - & 86 \\
\hline 43 & 102 & 100 & - & 97 & 101 & 101 & 104 & 100 & - & - & 97 & 99 \\
\hline 45 & - & - & - & - & - & - & 129 & - & - & - & - & 133 \\
\hline 46 & - & - & - & - & - & - & 99 & 91 & - & - & 92 & 88 \\
\hline 47 & - & - & - & - & - & - & 116 & 105 & - & - & - & 93 \\
\hline 48 & - & - & - & - & - & - & 70 & 63 & - & - & 60 & 58 \\
\hline 51 & 42 & - & - & 40 & - & - & 42 & 40 & - & - & 39 & 39 \\
\hline 52 & 35 & - & - & 36 & - & - & 33 & 34 & - & - & 30 & 33 \\
\hline 54 & - & - & - & - & - & - & 24 & 24 & - & - & 23 & 23 \\
\hline 55 & - & - & - & - & - & - & 51 & 48 & - & - & 44 & 48 \\
\hline 62 & - & - & - & - & - & - & 42 & - & - & - & - & - \\
\hline 63 & - & - & - & - & - & - & - & - & - & - & - & - \\
\hline 65 & - & - & 118 & 116 & - & - & 125 & - & - & - & - & 123 \\
\hline 66 & 100 & - & 94 & 86 & 95 & - & 99 & 96 & - & 91 & - & 86 \\
\hline 69 & 34 & - & 26 & - & 34 & - & 33 & 26 & - & 29 & 28 & - \\
\hline 70 & - & 49 & 52 & 59 & 55 & - & 61 & 56 & 56 & 54 & - & 54 \\
\hline 71 & 30 & 27 & 30 & 27 & 28 & - & 34 & 33 & 31 & 29 & - & 29 \\
\hline $8: 1$ & 67.20 & - & - & 81.03 & - & - & 72.25 & - & 76.22 & - & - & 78.57 \\
\hline $17: 1$ & - & - & - & - & - & - & 71.20 & - & - & - & - & 63.19 \\
\hline $17: 8$ & - & - & - & - & - & - & 98.55 & - & - & - & - & 80.42 \\
\hline $20: 1$ & - & - & - & 59.77 & - & 59.67 & 58.64 & - & - & - & - & 54.95 \\
\hline $20: 8$ & - & - & - & 73.76 & - & - & 81.16 & - & - & - & - & 69.93 \\
\hline $9: 8$ & 74.40 & - & - & 63.12 & - & - & 68.84 & - & 65.96 & - & - & 65.73 \\
\hline $47: 45$ & - & - & - & - & - & - & 89.92 & - & - & - & - & 69.92 \\
\hline $48: 45$ & - & - & - & - & - & - & 54.26 & - & - & - & - & 43.61 \\
\hline $52: 51$ & 83.33 & - & - & 90.00 & - & - & 78.57 & 85.00 & - & - & 76.92 & 84.62 \\
\hline $54: 55$ & - & - & - & - & - & - & 47.06 & 50.00 & - & - & 52.27 & 47.92 \\
\hline $63: 62$ & - & - & - & - & - & - & - & - & - & - & - & - \\
\hline
\end{tabular}


Table 6.

Female cranial measurements and indices in the population of Somogyszil-Dögkút (cont'd)

\begin{tabular}{|c|c|c|c|c|c|c|c|c|c|c|c|c|}
\hline Martin & Grave I & & & & & & & & & & & \\
\hline No. & 67 & 68 & 70 & 71 & 75 & 92 & 93 & 108 & 109 & 115 & 118 & 123 \\
\hline 1 & 163 & 186 & 168 & - & 167 & - & 168 & 168 & 179 & - & - & 180 \\
\hline 5 & - & - & - & - & - & - & - & - & 105 & - & - & - \\
\hline 8 & - & 142 & 150 & 141 & - & - & - & 143 & 134 & - & - & 138 \\
\hline 9 & 88 & 98 & 96 & 100 & - & - & 95 & 95 & 98 & 85 & - & 95 \\
\hline 10 & - & - & 129 & - & - & - & - & 122 & - & 112 & - & 117 \\
\hline 11 & - & 128 & 126 & - & - & - & - & 121 & 118 & - & - & - \\
\hline 12 & - & 116 & 107 & - & - & - & 107 & 112 & 104 & - & - & 106 \\
\hline 17 & - & - & - & - & - & - & - & - & 134 & - & - & - \\
\hline 20 & - & 110 & 120 & - & - & - & - & 115 & 116 & - & - & - \\
\hline 38 & - & 1386 & 1430 & - & - & - & - & 1332 & 1340 & - & - & - \\
\hline 40 & - & - & - & - & - & - & - & - & 101 & - & - & - \\
\hline 43 & 93 & 105 & 105 & 105 & - & - & 99 & 105 & - & 95 & - & - \\
\hline 45 & - & - & - & - & - & - & - & - & 129 & - & - & - \\
\hline 46 & - & - & - & - & - & - & - & - & - & - & - & - \\
\hline 47 & - & - & - & - & - & - & - & - & 118 & - & - & - \\
\hline 48 & 64 & - & - & - & - & - & - & - & 74 & - & - & - \\
\hline 51 & 39 & 41 & 43 & 42 & - & - & - & - & 42 & - & 41 & - \\
\hline 52 & 32 & 35 & 33 & 33 & - & - & - & - & 34 & - & 35 & - \\
\hline 54 & - & - & - & - & - & - & - & - & 25 & - & - & - \\
\hline 55 & 46 & - & - & - & - & - & - & - & 52 & - & - & - \\
\hline 62 & - & - & - & - & - & - & - & - & 43 & - & - & - \\
\hline 63 & - & - & - & - & - & - & - & - & 40 & - & - & - \\
\hline 65 & - & 122 & - & - & - & - & 121 & 122 & - & - & - & - \\
\hline 66 & - & 99 & 95 & - & - & - & 96 & 88 & - & - & - & - \\
\hline 69 & 26 & 33 & - & 30 & - & 29 & 32 & 32 & 32 & 26 & 25 & - \\
\hline 70 & 55 & 62 & 61 & - & - & - & 59 & 54 & 55 & - & 52 & 53 \\
\hline 71 & 30 & 33 & 33 & 32 & - & 31 & 34 & 31 & 34 & - & 29 & 33 \\
\hline $8: 1$ & - & 76.34 & 89.29 & - & - & - & - & 85.12 & 74.86 & - & - & 76.67 \\
\hline $17: 1$ & - & - & - & - & - & - & - & - & 74.86 & - & - & - \\
\hline $17: 8$ & - & - & - & - & - & - & - & - & 100.00 & - & - & - \\
\hline $20: 1$ & - & 59.14 & 71.43 & - & - & - & - & 68.45 & 64.80 & - & - & - \\
\hline $20: 8$ & - & 77.46 & 80.00 & - & - & - & - & 80.42 & 86.57 & - & - & - \\
\hline $9: 8$ & - & 69.01 & 64.00 & 70.92 & - & - & - & 66.43 & 73.13 & - & - & 68.84 \\
\hline $47: 45$ & - & - & - & - & - & - & - & - & 91.47 & - & - & - \\
\hline $48: 45$ & - & - & - & - & - & - & - & - & 57.36 & - & - & - \\
\hline $52: 51$ & 82.05 & 85.37 & 76.74 & 78.57 & - & - & - & - & 80.95 & - & 85.37 & - \\
\hline $54: 55$ & - & - & - & - & - & - & - & - & 48.08 & - & - & - \\
\hline $63: 62$ & - & - & - & - & - & - & - & - & 93.02 & - & - & - \\
\hline
\end{tabular}


Table 6.

Female cranial measurements and indices in the population of Somogyszil-Dögkút (cont'd)

\begin{tabular}{|c|c|c|c|c|c|c|c|c|c|c|}
\hline \multirow{2}{*}{$\begin{array}{l}\text { Martin } \\
\text { No. }\end{array}$} & \multicolumn{10}{|c|}{ Grave No. } \\
\hline & 127 & 131 & $\begin{array}{l}\text { stray } \\
\text { finds }\end{array}$ & 132 & 133 & 137 & 141 & 143 & 145 & 148 \\
\hline 1 & - & 184 & 177 & - & 178 & 181 & 179 & - & 169 & 184 \\
\hline 5 & - & - & 93 & - & - & - & - & - & - & 103 \\
\hline 8 & - & - & 128 & - & - & 140 & 134 & - & 136 & 134 \\
\hline 9 & 95 & 96 & 92 & 98 & 95 & 88 & 96 & - & 91 & 87 \\
\hline 10 & - & - & 108 & - & - & - & 119 & - & 109 & 113 \\
\hline 11 & - & - & 114 & - & - & 121 & 123 & - & 125 & 118 \\
\hline 12 & - & - & 103 & - & - & 109 & 109 & - & - & 102 \\
\hline 17 & - & - & 128 & - & - & - & - & - & - & 127 \\
\hline 20 & - & - & 112 & - & - & 113 & 104 & - & 111 & 110 \\
\hline 38 & - & - & 1248 & - & - & 1370 & 1232 & - & 1253 & 1313 \\
\hline 40 & - & - & - & - & - & - & - & - & - & 98 \\
\hline 43 & - & 105 & 100 & - & 106 & 94 & 103 & - & 97 & 98 \\
\hline 45 & - & - & 125 & - & - & 124 & - & - & 131 & 122 \\
\hline 46 & - & - & 92 & - & 88 & 82 & - & - & 93 & 90 \\
\hline 47 & - & - & 110 & - & - & 108 & - & - & 99 & 107 \\
\hline 48 & - & - & 66 & - & - & 63 & - & - & 62 & 66 \\
\hline 51 & - & - & 41 & - & - & 39 & - & - & 41 & 40 \\
\hline 52 & - & - & 35 & - & - & 32 & - & - & 30 & 31 \\
\hline 54 & - & - & 23 & - & 25 & 23 & - & - & 25 & 26 \\
\hline 55 & - & - & 53 & - & - & 47 & - & - & 45 & 47 \\
\hline 62 & - & - & 42 & - & - & - & - & - & - & 44 \\
\hline 63 & - & - & - & - & 35 & 37 & - & - & 46 & 43 \\
\hline 65 & - & - & 111 & - & - & - & - & - & 117 & 113 \\
\hline 66 & - & 98 & 97 & - & - & 90 & 90 & 103 & 99 & 99 \\
\hline 69 & - & - & 30 & - & 29 & 31 & - & 31 & 26 & 29 \\
\hline 70 & 56 & 62 & 63 & 55 & 67 & 59 & 54 & 58 & 58 & 52 \\
\hline 71 & 29 & 36 & 30 & 29 & 33 & 30 & 35 & 27 & 29 & 33 \\
\hline $8: 1$ & - & - & 72.32 & - & - & 77.35 & 74.86 & - & 80.47 & 72.83 \\
\hline $17: 1$ & - & - & 72.32 & - & - & - & - & - & - & 69.02 \\
\hline $17: 8$ & - & - & 100.00 & - & - & - & - & - & - & 94.78 \\
\hline $20: 1$ & - & - & 63.28 & - & - & 62.43 & 58.10 & - & 65.68 & 59.78 \\
\hline $20: 8$ & - & - & 87.50 & - & - & 80.71 & 77.61 & - & 81.62 & 82.09 \\
\hline $9: 8$ & - & - & 71.88 & - & - & 62.86 & 71.64 & - & 66.91 & 64.93 \\
\hline $47: 45$ & - & - & 88.00 & - & - & 87.10 & - & - & 75.57 & 87.70 \\
\hline $48: 45$ & - & - & 52.80 & - & - & 50.81 & - & - & 47.33 & 54.10 \\
\hline $52: 51$ & - & - & 85.37 & - & - & 82.05 & - & - & 73.17 & 77.50 \\
\hline $54: 55$ & - & - & 43.40 & - & - & 48.94 & - & - & 55.56 & 55.32 \\
\hline $63: 62$ & - & - & - & - & - & - & - & - & - & 97.73 \\
\hline
\end{tabular}


Table 7.

Summary statistics of cranial measurements and indices in the population of Somogyszil-Dögkút

\begin{tabular}{|c|c|c|c|c|c|c|c|c|c|c|c|c|}
\hline Martin & Male & & & & & & Fema & les & & & & \\
\hline No. & $\mathbf{N}$ & Vmax & Vmin & $\mathbf{M}$ & SD & S.R. & $\mathbf{N}$ & Vmax & Vmin & $\mathbf{M}$ & SD & S.R. \\
\hline 1 & 21 & 197 & 170 & 185.43 & 7.25 & 118.85 & 22 & 191 & 163 & 178.00 & 7.76 & 133.79 \\
\hline 5 & 7 & 106 & 95 & 100.86 & 4.02 & 98.05 & 5 & 105 & 93 & 99.20 & 5.76 & 147.69 \\
\hline 8 & 19 & 152 & 133 & 140.58 & 6.19 & 123.8 & 16 & 150 & 125 & 138.00 & 6.12 & 127.50 \\
\hline 9 & 22 & 105 & 89 & 96.82 & 4.47 & 101.59 & 30 & 100 & 85 & 93.40 & 3.87 & 90.00 \\
\hline 10 & 16 & 133 & 109 & 119.25 & 5.30 & 110.42 & 15 & 129 & 108 & 116.93 & 5.71 & 124.13 \\
\hline 11 & 15 & 135 & 115 & 124.47 & 5.21 & 108.54 & 12 & 128 & 114 & 121.92 & 4.27 & 92.83 \\
\hline 12 & 17 & 122 & 103 & 112.06 & 5.25 & 116.67 & 15 & 116 & 102 & 108.20 & 4.09 & 95.12 \\
\hline 17 & 6 & 140 & 128 & 135.17 & 4.36 & 88.98 & 5 & 136 & 115 & 128.00 & 8.22 & 174.89 \\
\hline 20 & 14 & 122 & 106 & 113.21 & 4.32 & 108.00 & 12 & 120 & 100 & 110.58 & 5.62 & 147.89 \\
\hline 38 & 13 & 1620.61 & 1330.85 & 1446.34 & 93.79 & 83.74 & 12 & 1430.40 & 1231.85 & 1319.51 & 67.93 & 67.59 \\
\hline 40 & 3 & 103 & 91 & 95.33 & 6.66 & 135.92 & 4 & 101 & 86 & 94.75 & 6.50 & 138.30 \\
\hline 43 & 23 & 115 & 98 & 105.74 & 4.38 & 113.77 & 23 & 106 & 93 & 100.48 & 3.87 & 106.03 \\
\hline 45 & 10 & 144 & 126 & 133.90 & 5.43 & 106.47 & 7 & 133 & 122 & 127.57 & 3.99 & 83.13 \\
\hline 46 & 7 & 103 & 91 & 96.57 & 4.08 & 86.81 & 9 & 99 & 82 & 90.56 & 4.59 & 103.15 \\
\hline 47 & 8 & 126 & 106 & 117.63 & 6.48 & 92.57 & 8 & 118 & 93 & 107.00 & 8.25 & 126.92 \\
\hline 48 & 10 & 79 & 63 & 70.60 & 4.60 & 112.20 & 10 & 74 & 58 & 64.60 & 4.70 & 123.68 \\
\hline 51 & 13 & 45 & 40 & 42.31 & 1.65 & 91.67 & 16 & 43 & 39 & 40.69 & 1.30 & 76.47 \\
\hline 52 & 11 & 36 & 29 & 32.27 & 2.37 & 124.74 & 16 & 36 & 30 & 33.19 & 1.83 & 96.32 \\
\hline 54 & 9 & 27 & 23 & 25.00 & 1.41 & 78.33 & 10 & 26 & 23 & 24.10 & 1.10 & 64.71 \\
\hline 55 & 9 & 56 & 44 & 50.56 & 3.54 & 122.07 & 10 & 53 & 44 & 48.10 & 3.00 & 111.11 \\
\hline 62 & 7 & 51 & 43 & 46.57 & 2.64 & 94.29 & 4 & 44 & 42 & 42.75 & 0.96 & 36.23 \\
\hline 63 & 4 & 45 & 42 & 43.25 & 1.26 & 47.55 & 5 & 46 & 35 & 40.20 & 4.44 & 174.12 \\
\hline 65 & 10 & 137 & 120 & 125.20 & 5.07 & 88.95 & 10 & 125 & 111 & 118.80 & 4.57 & 84.63 \\
\hline 66 & 13 & 115 & 98 & 105.62 & 4.54 & 72.06 & 19 & 103 & 86 & 94.79 & 4.97 & 85.69 \\
\hline 69 & 20 & 38 & 26 & 32.90 & 2.99 & 104.91 & 22 & 34 & 25 & 29.59 & 2.87 & 112.55 \\
\hline 70 & 26 & 74 & 52 & 63.62 & 5.73 & 116.94 & 27 & 67 & 49 & 56.70 & 4.11 & 93.41 \\
\hline 71 & 30 & 40 & 27 & 32.33 & 2.95 & 109.26 & 30 & 36 & 27 & 30.97 & 2.48 & 99.20 \\
\hline $8: 1$ & 18 & 83.98 & 69.04 & 75.32 & 3.69 & 115.31 & 15 & 89.29 & 67.20 & 77.03 & 5.45 & 170.31 \\
\hline $17: 1$ & 6 & 77.14 & 68.82 & 72.43 & 2.97 & 95.81 & 5 & 74.86 & 63.19 & 70.12 & 4.41 & 142.26 \\
\hline $17: 8$ & 6 & 102.21 & 94.33 & 97.97 & 3.08 & 70.00 & 5 & 100.00 & 80.42 & 94.75 & 8.29 & 188.41 \\
\hline $20: 1$ & 13 & 65.14 & 56.99 & 61.03 & 2.51 & 100.4 & 13 & 71.43 & 54.95 & 62.01 & 4.60 & 184.00 \\
\hline $20: 8$ & 13 & 87.41 & 73.03 & 80.99 & 4.36 & 132.12 & 12 & 87.50 & 69.93 & 79.90 & 4.87 & 147.58 \\
\hline $9: 8$ & 16 & 72.79 & 58.55 & 68.35 & 3.51 & 106.36 & 16 & 74.40 & 62.86 & 68.04 & 3.61 & 109.39 \\
\hline $47: 45$ & 6 & 92.37 & 79.70 & 87.05 & 5.27 & 99.43 & 7 & 91.47 & 69.92 & 84.24 & 8.15 & 153.77 \\
\hline $48: 45$ & 7 & 56.35 & 46.67 & 52.16 & 3.24 & 102.86 & 7 & 57.36 & 43.61 & 51.47 & 4.67 & 148.25 \\
\hline $52: 51$ & 11 & 85.00 & 66.67 & 76.11 & 6.02 & 120.40 & 16 & 90.00 & 73.17 & 81.60 & 4.39 & 87.80 \\
\hline $54: 55$ & 9 & 56.82 & 44.23 & 49.66 & 4.42 & 107.80 & 9 & 55.56 & 43.40 & 49.84 & 3.96 & 96.59 \\
\hline $63: 62$ & 3 & 104.65 & 89.36 & 95.83 & 7.91 & 113.00 & 2 & 97.73 & 93.02 & 95.38 & 3.33 & 47.57 \\
\hline
\end{tabular}




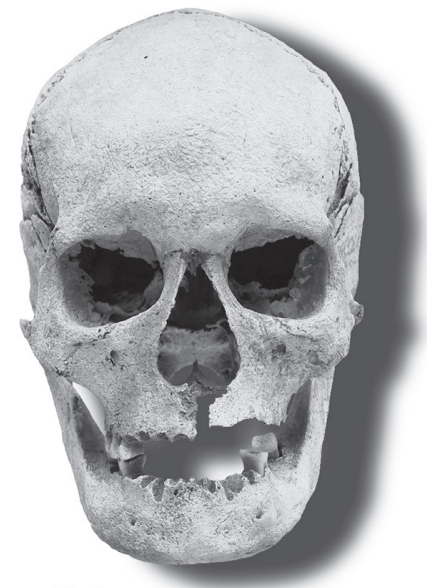

2A

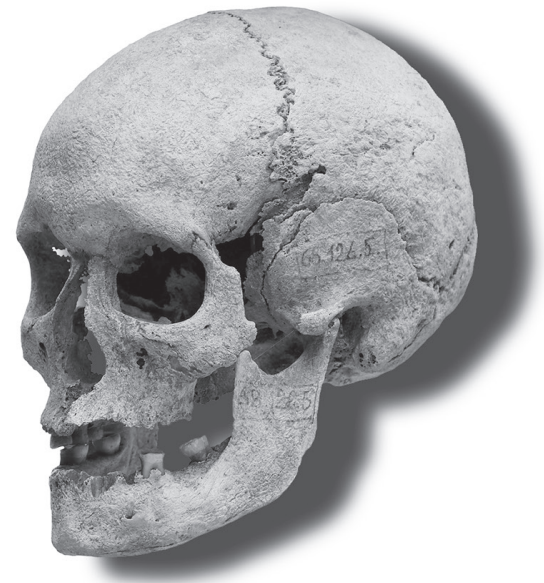

2B

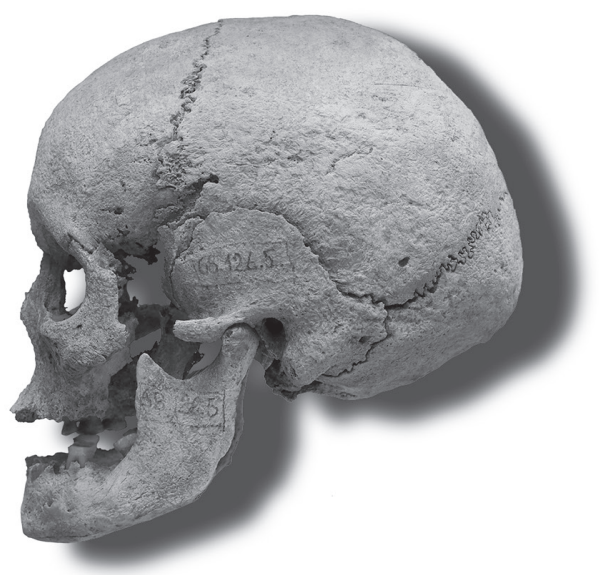

$2 \mathrm{C}$

Fig. 2. Grave no. 5a; adult male; skull - anterior (A), semi-profile (B) and lateral view (C)

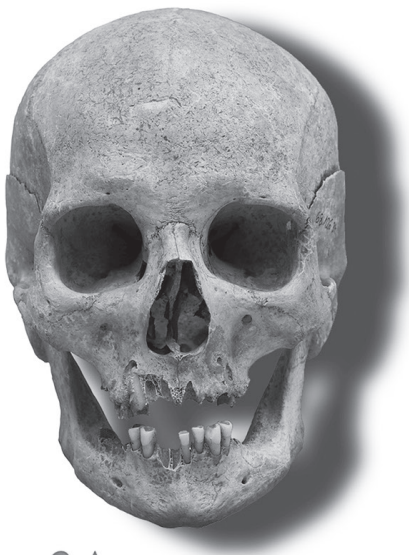

3A

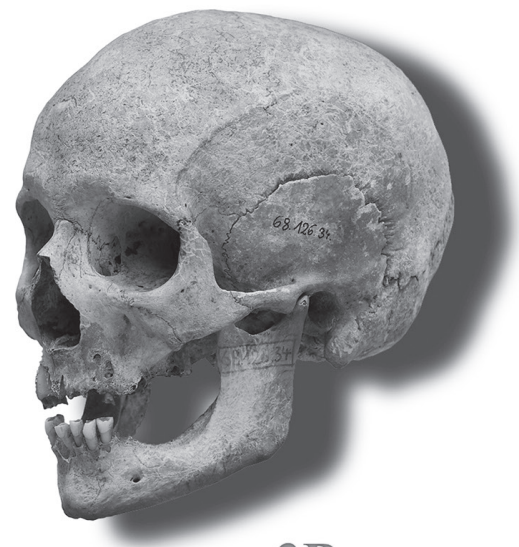

3B

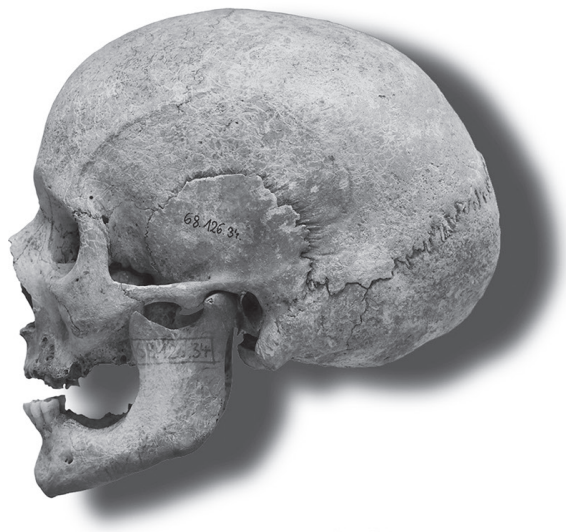

$3 \mathrm{C}$

Fig. 3. Grave no. 38; adult female; skull - anterior (A), semi-profile (B) and lateral view (C)

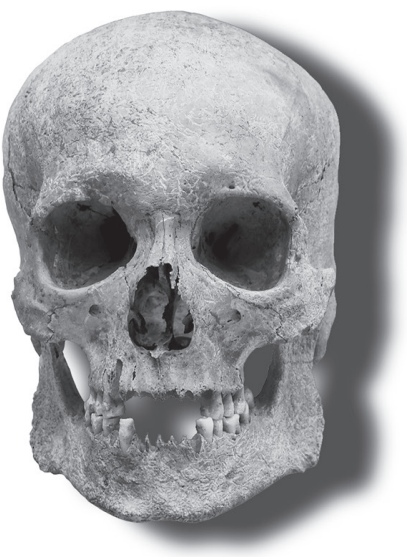

4A

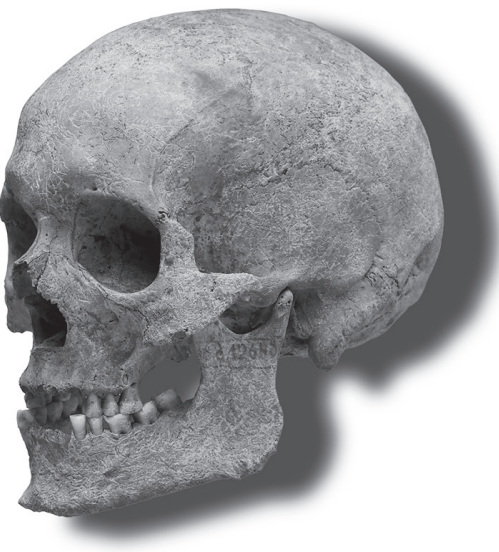

$4 \mathrm{~B}$

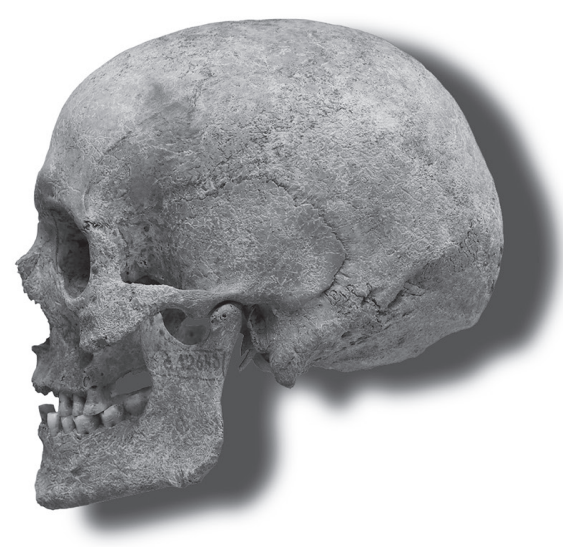

$4 \mathrm{C}$

Fig. 4. Grave no. 53; adult male; skull - anterior (A), semi-profile (B) and lateral view (C) 


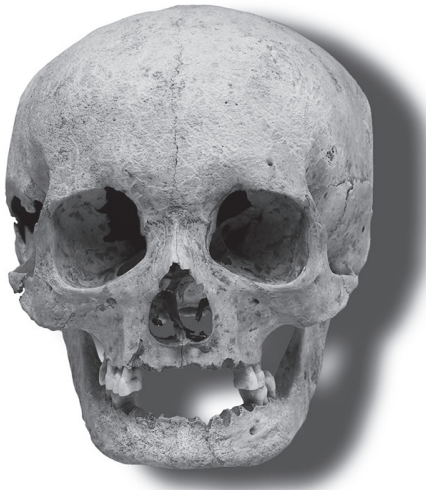

$5 \mathrm{~A}$

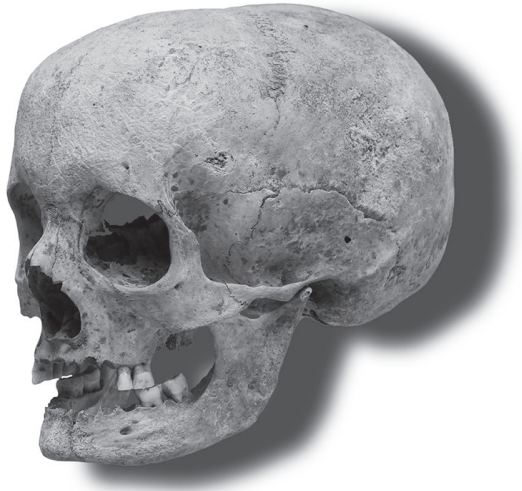

$5 \mathrm{~B}$

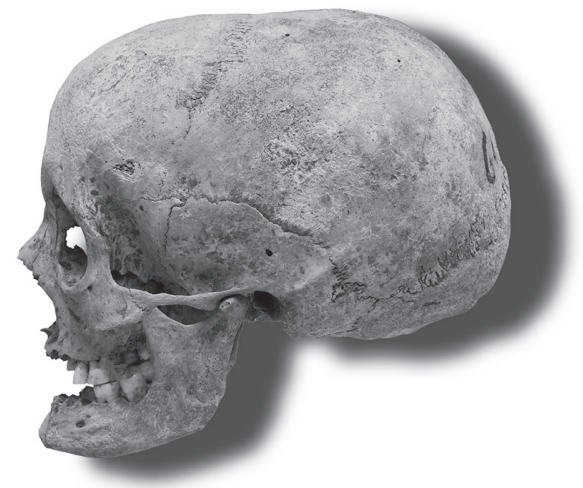

$5 \mathrm{C}$

Fig. 5. Grave no. 61; adult female; skull - anterior (A), semi-profile (B) and lateral view (C)

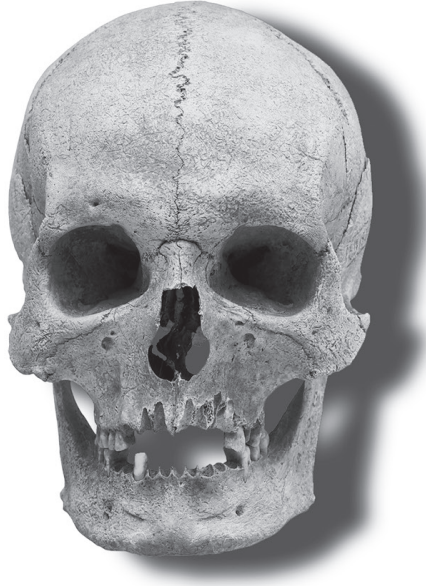

6A

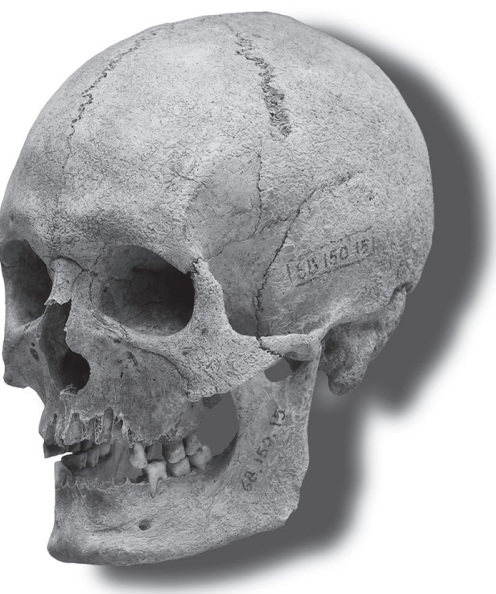

$6 \mathrm{~B}$

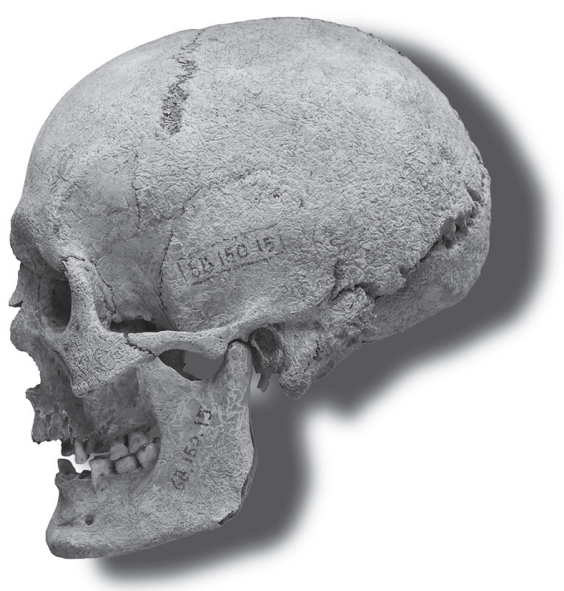

$6 \mathrm{C}$

Fig. 6. Grave no. 146; adult male; skull - anterior (A), semi-profile (B) and lateral view (C)

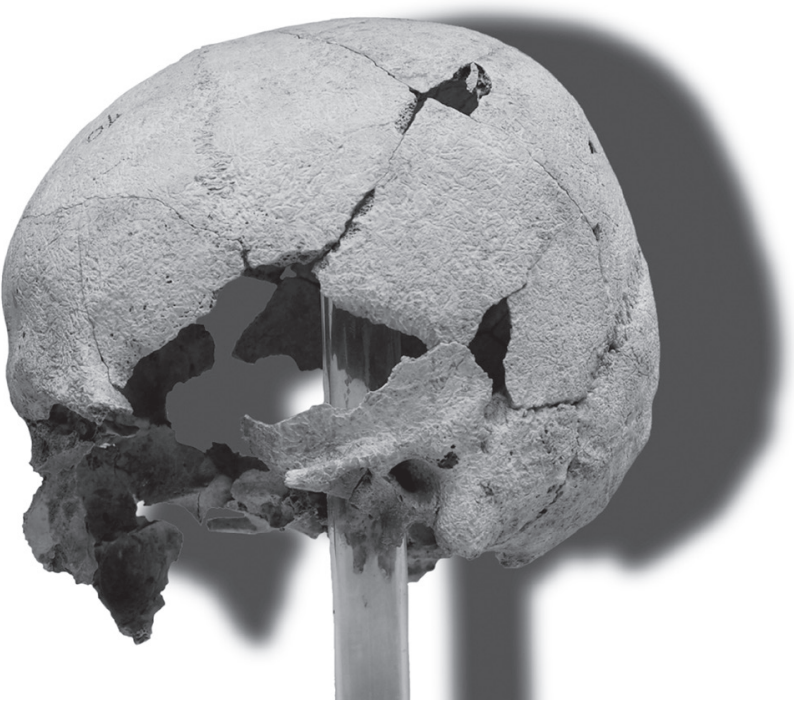

Fig. 7. Grave no. 70; adult female; skull - lateral view 
Table 8.

Distribution of cranial indices of the population of Somogyszil-Dögkút in the class categories of ALEKSEYEV-DEBETZ 1964

\begin{tabular}{|c|c|c|c|c|c|c|c|c|c|c|}
\hline \multirow{2}{*}{$\begin{array}{l}\text { Martin } \\
\text { No. }\end{array}$} & \multicolumn{2}{|l|}{ Class category } & \multicolumn{3}{|l|}{ Males } & \multicolumn{3}{|l|}{ Females } & \multicolumn{2}{|c|}{ Together } \\
\hline & & & & $\mathbf{N}$ & $\%$ & & $\mathbf{N}$ & $\%$ & $\mathbf{N}$ & $\%$ \\
\hline & \multicolumn{2}{|l|}{ Hyperdolichokran } & -73.2 & 5 & 27.78 & -74.1 & 4 & 26.67 & 9 & 27.27 \\
\hline \multirow[t]{5}{*}{$8: 1$} & Dolichokran & & $73.3-76.4$ & 6 & 33.33 & $74.2-77.3$ & 6 & 40.00 & 12 & 36.36 \\
\hline & Mesokran & & $76.5-79.9$ & 5 & 27.78 & $77.4-80.8$ & 2 & 13.33 & 7 & 21.21 \\
\hline & Brachykran & & $80.0-83.1$ & 1 & 5.56 & $80.9-84.0$ & 1 & 6.67 & 2 & 6.06 \\
\hline & \multicolumn{2}{|l|}{ Hyperbrachykran } & $83.2-$ & 1 & 5.56 & $84.1-$ & 2 & 13.33 & 3 & 9.09 \\
\hline & & $\Sigma$ & & 18 & & & 15 & & 33 & \\
\hline \multirow[t]{7}{*}{$17: 1$} & Hyperchamaekran & & -69.2 & 1 & 16.67 & -69.4 & 2 & 40.00 & 3 & 27.27 \\
\hline & Chamaekran & & $69.3-72.3$ & 2 & 33.33 & $69.5-72.5$ & 2 & 40.00 & 4 & 36.36 \\
\hline & Orthokran & & $72.4-75.6$ & 2 & 33.33 & $72.6-75.8$ & 1 & 20.00 & 3 & 27.27 \\
\hline & Hypsikran & & $75.7-78.7$ & 1 & 16.67 & $75.9-78.9$ & 0 & 0.00 & 1 & 9.09 \\
\hline & \multicolumn{2}{|l|}{ Hyperhypsikran } & $78.8-$ & 0 & 0.00 & $79.0-$ & 0 & 0.00 & 0 & 0.00 \\
\hline & & $\Sigma$ & & 6 & & & 5 & & 11 & \\
\hline & Hyperchamaekran & & -59.4 & 5 & 38.46 & -59.6 & 4 & 30.77 & 9 & 34.62 \\
\hline \multirow[t]{6}{*}{$20: 1$} & Chamaekran & & $59.5-61.8$ & 3 & 23.08 & $59.7-62.0$ & 3 & 23.08 & 6 & 23.08 \\
\hline & Orthokran & & $61.9-64.7$ & 4 & 30.77 & $62.1-64.9$ & 3 & 23.08 & 7 & 26.92 \\
\hline & Hypsikran & & $64.8-67.1$ & 1 & 7.69 & $65.0-67.3$ & 1 & 7.69 & 2 & 7.69 \\
\hline & \multicolumn{2}{|l|}{ Hyperhypsikran } & $67.2-$ & 0 & 0.00 & $67.4-$ & 2 & 15.38 & 2 & 7.69 \\
\hline & & $\Sigma$ & & 13 & & & 13 & & 26 & \\
\hline & Hypertapeinokran & & -87.9 & 0 & 0.00 & -87.1 & 1 & 20.00 & 1 & 9.09 \\
\hline \multirow[t]{6}{*}{$17: 8$} & Tapeinokran & & $88.0-92.3$ & 0 & 0.00 & $87.2-91.4$ & 0 & 0.00 & 0 & 0.00 \\
\hline & Metriokran & & $92.4-97.0$ & 2 & 33.33 & $91.5-96.1$ & 1 & 20.00 & 3 & 27.27 \\
\hline & Akrokran & & 97.1-101.4 & 3 & 50.00 & $96.2-100.4$ & 3 & 60.00 & 6 & 54.55 \\
\hline & Hyperakrokran & & $101.5-$ & 1 & 16.67 & 100.4 & 0 & 0.00 & 1 & 9.09 \\
\hline & & $\Sigma$ & & 6 & & & 5 & & 11 & \\
\hline & Hypertapeinokran & & -75.8 & 1 & 7.69 & -75.1 & 2 & 16.67 & 3 & 12.00 \\
\hline \multirow[t]{6}{*}{$20: 8$} & Tapeinokran & & 75.9-78.9 & 4 & 30.77 & $75.2-78.2$ & 2 & 16.67 & 6 & 24.00 \\
\hline & Metriokran & & $79.0-82.8$ & 3 & 23.08 & $78.3-82.1$ & 6 & 50.00 & 9 & 36.00 \\
\hline & Akrokran & & $82.9-85.9$ & 4 & 30.77 & $82.2-85.2$ & 0 & 0.00 & 4 & 16.00 \\
\hline & Hyperakrokran & & $86.0-91.8$ & 1 & 7.69 & $85.3-91.0$ & 2 & 16.67 & 3 & 12.00 \\
\hline & & $\Sigma$ & & 13 & & & 12 & & 25 & \\
\hline & Hyperstenometop & & -62.7 & 1 & 6.25 & -63 & 1 & 6.25 & 2 & 6.25 \\
\hline \multirow[t]{5}{*}{$9: 8$} & Stenometop & & $62.8-66.0$ & 2 & 12.50 & $63.1-66.3$ & 5 & 31.25 & 7 & 21.88 \\
\hline & Metriometop & & $66.1-69.6$ & 6 & 37.50 & $66.4-69.9$ & 5 & 31.25 & 11 & 34.38 \\
\hline & Eurymetop & & $69.7-72.9$ & 7 & 43.75 & $70.0-73.2$ & 4 & 25.00 & 11 & 34.38 \\
\hline & Hypereurymetop & & $73.0-$ & 0 & 0.00 & $73.3-$ & 1 & 6.25 & 1 & 3.13 \\
\hline & & $\Sigma$ & & 16 & & & 16 & & 32 & \\
\hline
\end{tabular}


Table 8.

Distribution of cranial indices of the population of Somogyszil-Dögkút in the class categories of ALEKSEYEv-DEBETZ 1964 (cont'd)

\begin{tabular}{|c|c|c|c|c|c|c|c|c|c|c|}
\hline \multirow{2}{*}{$\begin{array}{l}\text { Martin } \\
\text { No. }\end{array}$} & \multicolumn{2}{|l|}{ Class category } & \multicolumn{3}{|l|}{ Males } & \multicolumn{3}{|l|}{ Females } & \multicolumn{2}{|c|}{ Together } \\
\hline & & & & $\mathbf{N}$ & $\%$ & & $\mathbf{N}$ & $\%$ & $\mathbf{N}$ & $\%$ \\
\hline & Hyperoligenkephal & & -1227 & 0 & 0.00 & -1096 & 0 & 0.00 & 0 & 0.00 \\
\hline & Oligenkephal & & $1228-1337$ & 1 & 7.69 & $1097-1195$ & 0 & 0.00 & 1 & 4.00 \\
\hline \multirow[t]{5}{*}{38} & Euenkephal & & $1338-1462$ & 6 & 46.15 & 1196-1307 & 5 & 41.67 & 11 & 44.00 \\
\hline & Aristenkephal & & $1463-1572$ & 5 & 38.46 & $1308-1406$ & 6 & 50.00 & 11 & 44.00 \\
\hline & Hyperaristenkephal & & 1573 & 1 & 7.69 & $1407-1582$ & 1 & 8.33 & 2 & 8.00 \\
\hline & & $\Sigma$ & & 13 & & & 12 & & 25 & \\
\hline & Hypereuryprosop & & -80.5 & 1 & 16.67 & -80.1 & 2 & 33.33 & 3 & 25.00 \\
\hline \multirow[t]{6}{*}{$47: 45$} & Euryprosop & & $80.6-85.8$ & 1 & 16.67 & $80.2-85.4$ & 0 & 0.00 & 1 & 8.33 \\
\hline & Mesoprosop & & 85.9-91.6 & 3 & 50.00 & $85.5-91.1$ & 4 & 66.67 & 7 & 58.33 \\
\hline & Leptoprosop & & 91.7-96.9 & 1 & 16.67 & 91.2-96.4 & 0 & 0.00 & 1 & 8.33 \\
\hline & Hyperleptoprosop & & $97.0-$ & 0 & 0.00 & $96.5-$ & 0 & 0.00 & 0 & 0.00 \\
\hline & & $\Sigma$ & & 6 & & & 6 & & 12 & \\
\hline & Hypereuryen & & -48.3 & 1 & 14.29 & -48.1 & 2 & 28.57 & 3 & 21.43 \\
\hline \multirow[t]{6}{*}{$48: 45$} & Euryen & & $48.4-51.4$ & 2 & 28.57 & $48.2-51.2$ & 1 & 14.29 & 3 & 21.43 \\
\hline & Mesen & & $51.5-54.9$ & 3 & 42.86 & $51.3-54.7$ & 3 & 42.86 & 6 & 42.86 \\
\hline & Lepten & & $55.0-58.0$ & 1 & 14.29 & $54.8-57.8$ & 1 & 14.29 & 2 & 14.29 \\
\hline & Hyperlepten & & $58.1-$ & 0 & 0.00 & $57.9-$ & 0 & 0.00 & 0 & 0.00 \\
\hline & & $\Sigma$ & & 7 & & & 7 & & 14 & \\
\hline & Hyperchamaekonch & & -73.8 & 5 & 45.45 & -76.4 & 1 & 6.25 & 6 & 22.22 \\
\hline \multirow[t]{6}{*}{$52: 51$} & Chamaekonch & & 73.9-78.7 & 3 & 27.27 & $76.5-81.5$ & 6 & 37.50 & 9 & 33.33 \\
\hline & Mesokonch & & $78.8-84.3$ & 2 & 18.18 & $81.6-87.3$ & 8 & 50.00 & 10 & 37.04 \\
\hline & Hypsikonch & & $84.4-89.2$ & 1 & 9.09 & $87.4-92.4$ & 1 & 6.25 & 2 & 7.41 \\
\hline & Hyperhypsikonch & & $89.3-$ & 0 & 0.00 & $92.5-$ & 0 & 0.00 & 0 & 0.00 \\
\hline & & $\Sigma$ & & 11 & & & 16 & & 27 & \\
\hline & Hyperleptorrhin & & -42.5 & 0 & 0.00 & -43.3 & 0 & 0.00 & 0 & 0.00 \\
\hline \multirow[t]{6}{*}{$54: 55$} & Leptorrhin & & $42.6-46.6$ & 3 & 33.33 & $43.4-47.5$ & 2 & 22.22 & 5 & 27.78 \\
\hline & Mesorrhin & & $46.7-51.1$ & 4 & 44.44 & $47.6-52.1$ & 4 & 44.44 & 8 & 44.44 \\
\hline & Chamaerrhin & & $51.2-55.2$ & 0 & 0.00 & $52.2-56.3$ & 3 & 33.33 & 3 & 16.67 \\
\hline & Hyperchamaerrhin & & $55.3-$ & 2 & 22.22 & $56.4-$ & 0 & 0.00 & 2 & 11.11 \\
\hline & & $\Sigma$ & & 9 & & & 9 & & 18 & \\
\hline & Hyperleptostaphylin & & -75.7 & 0 & 0.00 & -75.8 & 0 & 0.00 & 0 & 0.00 \\
\hline \multirow[t]{5}{*}{$63: 62$} & \multicolumn{2}{|l|}{ Leptostaphylin } & $75,8-82,6$ & 0 & 0.00 & $75.9-82.7$ & 0 & 0.00 & 0 & 0.00 \\
\hline & \multicolumn{2}{|l|}{ Mesostaphylin } & $82,7-90,3$ & 1 & 33.33 & $82.8-90.5$ & 0 & 0.00 & 1 & 20.00 \\
\hline & \multicolumn{2}{|l|}{ Brachystaphylin } & $90,4-97,2$ & 1 & 33.33 & $90.6-97.4$ & 1 & 50.00 & 2 & 40.00 \\
\hline & Hyperbrachystaphylin & & $97.3-$ & 1 & 33.33 & $97.5-$ & 1 & 50.00 & 2 & 40.00 \\
\hline & & $\Sigma$ & & 3 & & & 2 & & 5 & \\
\hline
\end{tabular}


a merely hypothetical proposal - it should here be recalled that during this period brachycranic skulls were frequent among populations of presumably Sarmatian origin and/or populations with a significant Sarmatian component.

\section{Metric characteristics of the postcranial skeleton, estimated stature}

The individual postcranial measurements and the estimated stature are presented in Tables 9-10. The mean height of males is $165.94 \mathrm{~cm}$, while it is $156.86 \mathrm{~cm}$ for females. Taking a look at the $\mathrm{N}-\mathrm{S} / \mathrm{S}-\mathrm{N}$ oriented burials, it can be seen that three males from those graves were suitable for stature calculation (Grave no. 18: $171.33 \mathrm{~cm}$; Grave no. 41: $166.05 \mathrm{~cm}$; Grave no. 50: $174.33 \mathrm{~cm}$ ). If they are excluded from the stature mean calculation, the average height of males lowers to $165.31 \mathrm{~cm}$. Two of these three males (Grave no. 18 and Grave no. 50) had a stature significantly exceeding this mean value. Although far-reaching conclusions can hardly be drawn owing to the extremely low number of cases, if this record is viewed together with the data on skulls, it indirectly underpins the assumption that the $\mathrm{N}-\mathrm{S} / \mathrm{S}-\mathrm{N}$ oriented graves contained the burials of an immigrant group that in terms of certain anthropological traits differed substantially from the overwhelming majority of the community using the cemetery.

The average stature (calculated from the lower limb bones) of the population of the Carpathian Basin during the Roman Age was $166.09 \mathrm{~cm}$ for males, and $156.91 \mathrm{~cm}$ for females. ${ }^{27}$ The average height of the SomogyszilDögkúti dủlő population fits nicely into this picture.

\section{Traumas and injuries}

The frequency of fractures and other injuries caused by accidents or interpersonal violence is fairly low in the Somogyszil-Dögkúti dűlő population. Fracture of the lower limb was identified in one case: a healed fracture was found in the proximal third of the right fibula of an adult woman interred in Grave no. 16. Healed fractures of the upper limb was noted in three cases: a woman buried in Grave no. 20 had broken her right ulna near the distal end, a mid diaphyseal fracture healed with an angulation was observed on the left radius of the man interred in Grave no. 23 (Fig. 8), and a mature man in Grave no. 41 had broken his left radius in the distal third. In addition, one other bone trauma was found: an $\sim 11 \mathrm{~mm}$ long healed depressed fracture probably caused by a moderately sharp implement was identified on the left parietal bone of an elderly woman from Grave no. 19 (Fig. 9).

\section{Biological distance between Somogyszil-Dögkúti dülö and other archaeological populations}

The pairwise Penrose distances $\left(\mathrm{C}_{\mathrm{R}}{ }^{2}\right.$ : "size" and "shape" combined) between the Somogyszil-Dögkúti dülö and other male cranial series are presented in Table 11.

From the comparative male cranial series below the $1 \%$ error band $\left(\mathrm{C}_{\mathrm{R}}{ }^{2} \leq 0.196\right)$ are the late Roman period sites of Esztergom-Bánomi dülő $\left(\mathrm{C}_{\mathrm{R}}{ }^{2}=0.096\right)$, Keszthely-Dobogó $\left(\mathrm{C}_{\mathrm{R}}{ }^{2}=0.183\right)$ and Tác-Margittelep $\left(\mathrm{C}_{\mathrm{R}}{ }^{2}=0.188\right)$, the Avar period series of Kaposvarr-Road 61, Site $26\left(\mathrm{C}_{\mathrm{R}}{ }^{2}=0.054\right)$, Toponár-40-es örház [Toponár watchman's house No. 40] $\left(C_{R}^{2}=0.075\right)$, Zalakomár-Lesvári dülő II $\left(C_{R}^{2}=0.076\right)$, Kereki-Homokbánya $\left(C_{R}^{2}=0.100\right)$, KeszthelyVáros $\left(\mathrm{C}_{\mathrm{R}}{ }^{2}=0.121\right)$, Kaposvár-Fészerlakpuszta $\left(\mathrm{C}_{\mathrm{R}}{ }^{2}=0.148\right)$, Zelovce (Zsély) $\left(\mathrm{C}_{\mathrm{R}}{ }^{2}=0.154\right)$ and Virt $\left(\mathrm{C}_{\mathrm{R}}{ }^{2}=0.194\right)$. Cranial series below the $2 \%$ error band $\left(\mathrm{C}_{\mathrm{R}}{ }^{2}<0.235\right)$ are the Keszthely burial ground uncovered by Vilmos Lipp and assigned to the Keszthely culture $\left(\mathrm{C}_{\mathrm{R}}{ }^{2}=0.200\right)$, and the Late Avar period cemeteries of Tiszaderzs $\left(\mathrm{C}_{\mathrm{R}}{ }^{2}=0.213\right)$ and Nové Zámky (Érsekújvár) $\left(\mathrm{C}_{\mathrm{R}}{ }^{2}=0.229\right)$.

The biological distance-based relations of the close analogies of Somogyszil-Dögkúti dülő male cranial series is visualized by a dendrogram (Fig. 10). Accordingly, Somogyszil-Dögkúti düló clusters together with the Avar period populations of Kaposvár-Road 61, Site 26, Toponár 40-es őrház [Toponár watchman's house No. 40] and Kereki-Homokbánya. This joins together still within the $1 \%$ error band threshold value $(0.196)$ with an other cluster composed of the Avar period series of Kaposvár-Fészerlakpuszta and Zalakomár-Lesvári dűlő II, and the

\footnotetext{
${ }^{27}$ ÉRY 1998.
} 


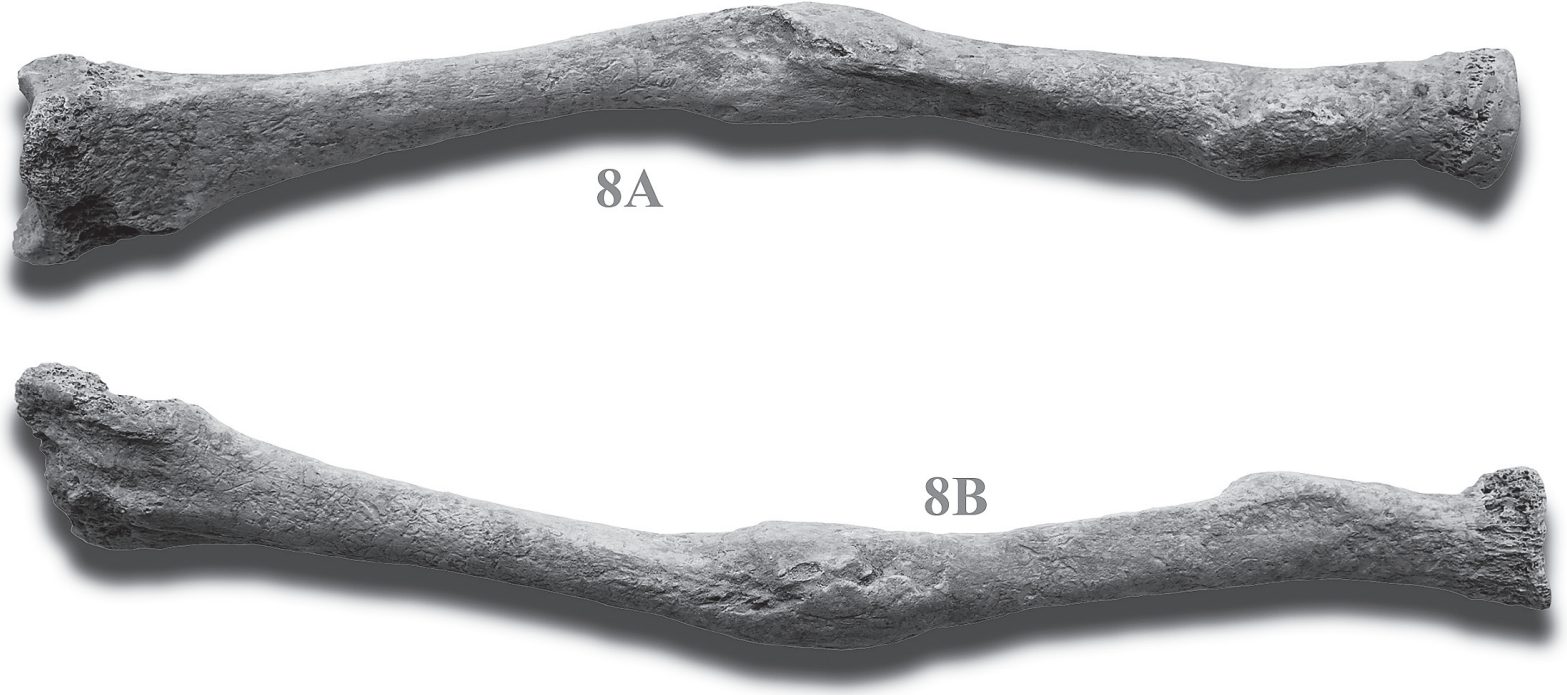

Fig. 8. Grave no. 23; adult male; fracture of the left radius healed with an angulation (A, B)

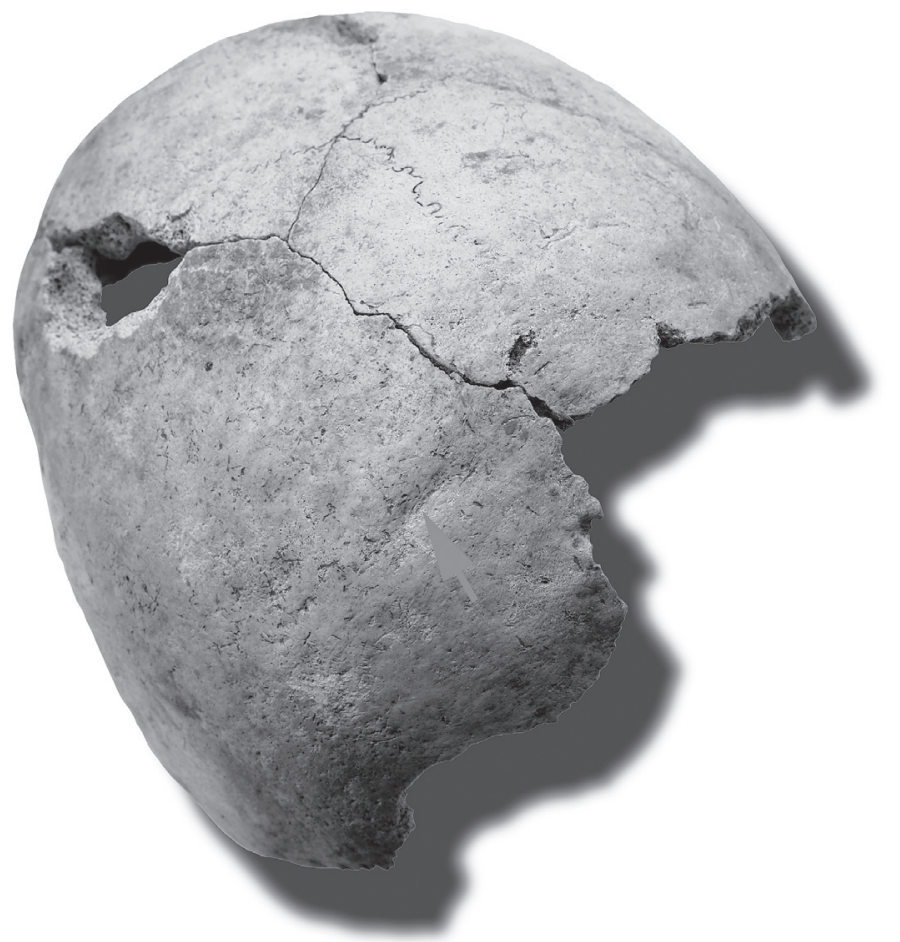

Fig. 9. Grave no. 19; senile female; healed depressed fracture on the left parietal bone 


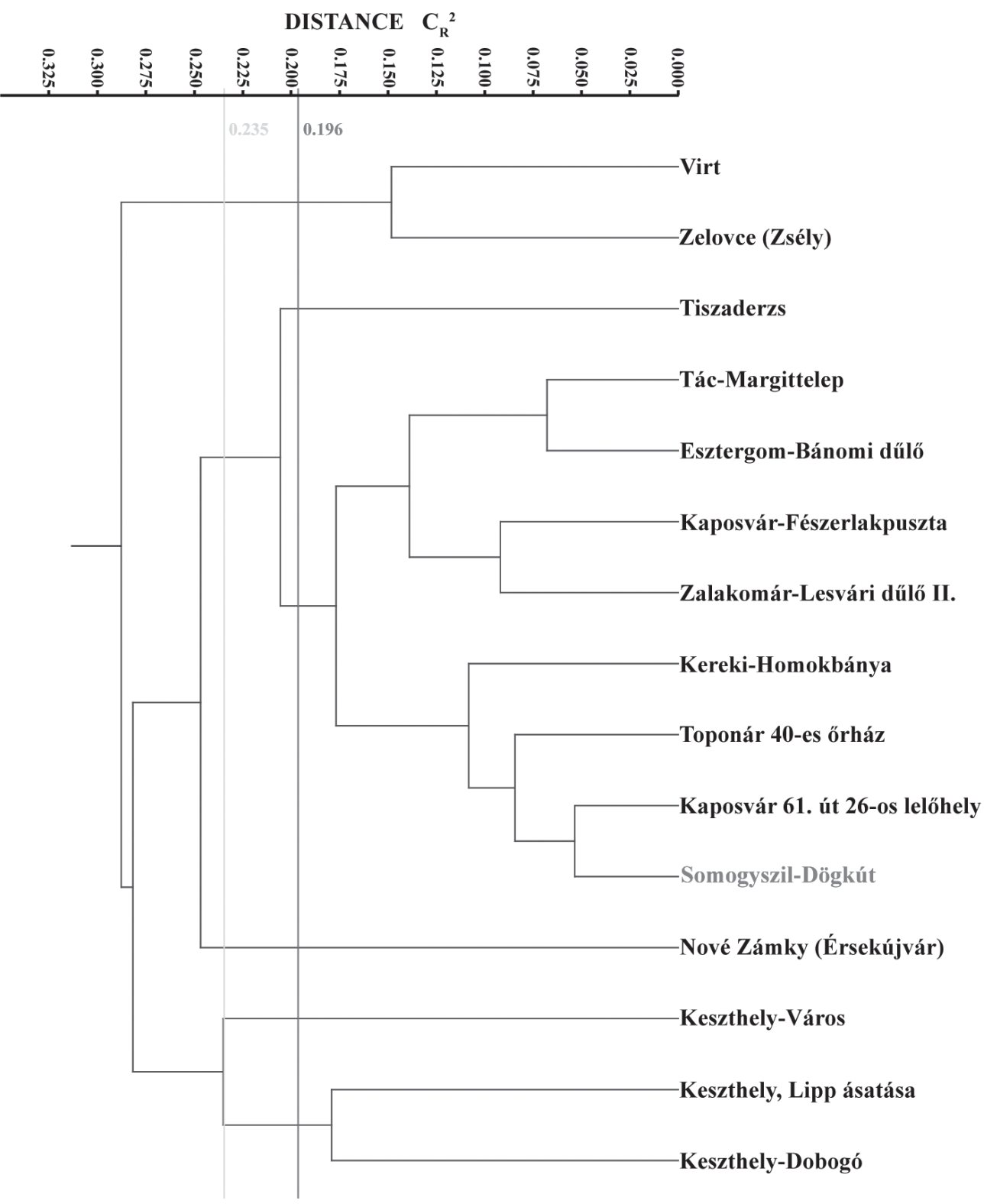

Fig. 10. Dendrogram showing the relations among the male cranial series of Somogyszil-Dögkút and its close (below the $2 \%$ or $1 \%$ error band) analogies - Penrose distance, UPGMA hierarchical clustering method 
Table 9.

The M1 measurement of long bones, and the calculated statures of the males of Somogyszil-Dögkút

\begin{tabular}{|c|c|c|c|c|c|c|c|c|c|c|c|c|c|}
\hline \multirow{2}{*}{$\begin{array}{l}\text { Grave } \\
\text { No. }\end{array}$} & \multicolumn{2}{|c|}{ Humerus } & \multicolumn{2}{|c|}{ Ulna } & \multicolumn{2}{|c|}{ Radius } & \multicolumn{2}{|c|}{ Femur } & \multicolumn{2}{|c|}{ Tibia } & \multicolumn{2}{|c|}{ Fibula } & \multirow{2}{*}{$\begin{array}{l}\text { Calculated stature } \\
(\mathrm{cm})\end{array}$} \\
\hline & Left & Right & Left & Right & Left & Right & Left & Right & Left & Right & Left & Right & \\
\hline 17 & & & & & & & 447 & 446 & 367 & & & & 166.86 \\
\hline 18 & 319 & & & & & & 463 & 463 & & 365 & & & 171.33 \\
\hline 27 & 316 & 305 & 259 & 258 & & & 424 & 426 & & 342 & & & 161.04 \\
\hline 29 & & & 272 & & & & 432 & 438 & & & & & 163.75 \\
\hline 33 & 309 & 303 & & & 233 & 232 & 421 & 422 & & 346 & & & 160.09 \\
\hline 39 & 300 & 295 & & & & & & & 343 & & & & 160.19 \\
\hline 41 & 315 & & & & & & 441 & 446 & 350 & & & & 166.05 \\
\hline 45 & 324 & & & & 241 & & & & 362 & & & & 166.44 \\
\hline 46 & 324 & & 260 & & 243 & & 433 & 423 & 349 & 339 & & & 161.85 \\
\hline 48 & & & & & 243 & 238 & 444 & & & & & & 166.18 \\
\hline 50 & 326 & & & & & & & & 386 & & & & 174.33 \\
\hline 51 & 324 & & 254 & 250 & 236 & 231 & 427 & & 345 & 346 & & & 161.58 \\
\hline 53 & 329 & 323 & 277 & 273 & 253 & 253 & 432 & & & & & & 162.93 \\
\hline 59 & & & & & 240 & & & & & & & & \\
\hline 60 & & & & & & & 456 & & & 376 & & & 169.44 \\
\hline 79 & & 315 & & & & & & 458 & & & & & 169.98 \\
\hline 84 & 339 & & & & 253 & & & & & & & & \\
\hline 86 & & & & & 234 & & & 432 & 353 & & & & 162.93 \\
\hline 103 & & & & & & & 452 & & & & & & 168.35 \\
\hline $113 a$ & & & & & 282 & & 440 & & & & & & 165.10 \\
\hline 116 & & 324 & & & & & & & & & & & \\
\hline 124 & & & & & & & 408 & 411 & & & & & 156.83 \\
\hline 130 & 334 & 323 & & 271 & & 247 & 452 & 450 & 379 & 382 & & & 168.08 \\
\hline 138 & & & & & & & & 469 & & & & & 172.96 \\
\hline 140 & & & & & & & 468 & 454 & & & & & 170.79 \\
\hline 144 & & & & & & & 452 & & & & & & 168.35 \\
\hline 146 & 323 & 317 & 277 & 279 & 249 & & 468 & 463 & 391 & 385 & & & 172.01 \\
\hline 147 & & 304 & & & & 225 & 425 & & & & & & 161.04 \\
\hline
\end{tabular}


Table 10.

The M1 measurement of long bones, and the calculated statures of the females of Somogyszil-Dögkút

\begin{tabular}{|c|c|c|c|c|c|c|c|c|c|c|c|c|c|}
\hline \multirow{2}{*}{$\begin{array}{l}\text { Grave } \\
\text { No. }\end{array}$} & \multicolumn{2}{|c|}{ Humerus } & \multicolumn{2}{|l|}{ Ulna } & \multicolumn{2}{|c|}{ Radius } & \multicolumn{2}{|c|}{ Femur } & \multicolumn{2}{|c|}{ Tibia } & \multicolumn{2}{|c|}{ Fibula } & \multirow{2}{*}{$\begin{array}{l}\text { Calculated stature } \\
(\mathrm{cm})\end{array}$} \\
\hline & Left & Right & Left & Right & Left & Right & Left & Right & Left & Right & Left & Right & \\
\hline 16 & 314 & 311 & & & & 225 & 421 & 423 & & & & & 160.22 \\
\hline 19 & & & & & & & & 393 & & & & & 152.36 \\
\hline 20 & & 288 & 243 & & & & 400 & 398 & & & & & 153.99 \\
\hline 21 & & & 244 & & & & 408 & & 340 & 338 & & & 156.43 \\
\hline 24 & & & & & & & 409 & & 325 & & & & 156.70 \\
\hline 26 & & & & & & & & & & & 325 & & \\
\hline 36 & & & 251 & & 228 & & & & & & & & \\
\hline 38 & 303 & 300 & & 225 & & & 437 & 439 & 356 & 356 & & & 163.61 \\
\hline 40 & 281 & & & & & & 405 & 404 & 323 & 323 & & & 155.48 \\
\hline 43 & 303 & 300 & & & & & 410 & 407 & 351 & 344 & & & 156.56 \\
\hline 44 & & 281 & & & & 203 & 398 & 392 & 310 & 306 & & & 152.91 \\
\hline 55 & & & & & & & 412 & 421 & & & & & 158.46 \\
\hline 61 & & & & 229 & 216 & 212 & & 415 & 333 & 331 & & & 158.33 \\
\hline \begin{tabular}{|l|}
89 \\
\end{tabular} & & & & & 203 & & 393 & & & 319 & & & 152.36 \\
\hline 92 & & & & 266 & & & & & & & & & \\
\hline \begin{tabular}{|l|}
97 \\
\end{tabular} & & 286 & & & & & & & & & & & \\
\hline 108 & & & & & & & 409 & 410 & 340 & 337 & & & 156.83 \\
\hline 109 & 296 & & & & & 219 & & & & & & & \\
\hline 115 & 266 & & & & & & & 378 & & & & & 148.30 \\
\hline \begin{tabular}{|l|}
127 \\
\end{tabular} & & & & & & & 419 & & & 327 & & & 159.41 \\
\hline 131 & 322 & & & & & & & & & & & & \\
\hline 133 & & & & & & & 431 & & & & & & 162.66 \\
\hline 143 & & & & & & & & 428 & & & & & 161.85 \\
\hline 145 & 301 & 297 & & & 219 & & 421 & 422 & 342 & & & & 160.09 \\
\hline 148 & 283 & 275 & 242 & 239 & 215 & 216 & 400 & 399 & 321 & 319 & 318 & 308 & 153.72 \\
\hline
\end{tabular}


Table 11.

Penrose distance between the male cranial series of Somogyszil-Dögkút and other comparative series

\begin{tabular}{|c|c|}
\hline Male cranial series & \begin{tabular}{|l|} 
PENROSE distance \\
(size and shape) C $^{\mathrm{R} 2}$
\end{tabular} \\
\hline \multicolumn{2}{|c|}{$*=$ below the $2 \%$ error band $; * * *=$ below the $1 \%$ error band } \\
\hline \multicolumn{2}{|l|}{ Carpathian Basin, $\sim 2^{\text {nd }}-5^{\text {th }}$ centuries } \\
\hline Budapest, III. kerület, Kaszás dűlő, Raktárrét (FRÁTER 1993) & 0.453 \\
\hline Esztergom-Bánomi dülő (MERCZI 2008) & $0.089 * * *$ \\
\hline Keszthely-Dobogó (VARGA et al. 2005) & $0.183 * * *$ \\
\hline Pécs (Geisler Eta utca 8. and 14., István tér 12.) (ÉRY 1973) & 0.240 \\
\hline Tác-Margittelep (ÉRY 2000) & $0.188 * * *$ \\
\hline Tokod (ÉRY 1981) & 0.348 \\
\hline Visegrád-Diós (MERCZI 2001) & 0.236 \\
\hline \multicolumn{2}{|l|}{ Carpathian Basin, $5^{\text {th }}-8^{\text {th }}$ centuries } \\
\hline Adorján-Ország (BARTUCZ-FARKAS 1957) & 0.449 \\
\hline Alattyán-Tulát (WENGER 1957) & 0.754 \\
\hline Ártánd-Kapitány (ÉRY 1966) & 0.308 \\
\hline Bačko Petrovo Selo (Péterréve) (ÉRY 1990) & 0.430 \\
\hline Bácska-Topolya (FARKAS-MARCSIK 1984) & 2.418 \\
\hline Bágyogszovát-Gyürhegy (DEzsö 1968) & 0.534 \\
\hline Csákberény-Orondpuszta (ÉRY 2001a) & 0.443 \\
\hline Elöszállás-Bajcsihegy (WENGER 1966) & 1.377 \\
\hline Gyenesdiás (T. RENDES-TótH 2000) & 0.611 \\
\hline Holiare (Gellér) (MALA cit. RösING-SCHWIDETZKY 1977) & 0.373 \\
\hline Jánoshida-Tótkérpuszta (WENGER 1953) & 1.021 \\
\hline Kassa-Zsebes (ThURZo 1984) & 0.251 \\
\hline Kaposvár 61-es út 26. lelöhely [Kaposvár, Road 61, Site 26] (based on the unpublished remeasurement data of ÉvINGER) (28 $^{28}$ & $0.054 * * *$ \\
\hline Kaposvár-Fészerlakpuszta (based on the unpublished remeasurement data of ÉVINGER) ${ }^{29}$ & $0.148 * * *$ \\
\hline Kecel I. (LIPTÁK 1954) & 0.715 \\
\hline Kereki-Homokbánya (based on the unpublished remeasurement data of ÉVINGER) $)^{30}$ & $0.100 * * *$ \\
\hline Keszthely, Lipp féle feltárás [V. Lipp’s excavation] (VARGA et al. 2003) & 0.200* \\
\hline Keszthely-Város (based on the unpublished remeasurement data of ÉvINGER) ${ }^{31}$ & $0.121 * * *$ \\
\hline Kékesd (WENGER 1968) & 0.700 \\
\hline Kiskőrös-Város (LiPTÁK 1983) & 0.526 \\
\hline Kiszombor (BARTUCZ 1936) & 0.680 \\
\hline Langobards - merged series (Hungary and Austria, $5^{\text {th }}-7^{\text {th }}$ centuries) (cit. RÖsING-SCHWIDETZKY 1977) & 0.242 \\
\hline Lesencetomaj (Bíró 1999) & 0.355 \\
\hline Loebersdorf (GREFEN-PETERS 1987) & 3.201 \\
\hline Madaras-Téglavető (LIPTÁK-MARCSIK 1976) & 1.289 \\
\hline Moravica (Bácskossuthfalva) (CZÉKus 1985) & 1.046 \\
\hline Nové Zámky (Érsekújvár) (VLADAROVA et al., cit. RöSING-SCHWIDETZKY 1977) & $0.229 *$ \\
\hline Pókaszepetk (based on the unpublished remeasurement data of ÉVINGER) ${ }^{32}$ & 0.604 \\
\hline Siófok-Kiliti (SusKoviCS 1993) & 0.599 \\
\hline Solymár (FERENCZ 1983) & 0.344 \\
\hline
\end{tabular}

${ }^{28}$ The original metric data providing publication: ÉvINGERBERNERT 2005.

${ }^{29}$ The original metric data providing publications: WENGER 1975, FóTHI 1988.

${ }^{30}$ The original metric data providing publication: BERNERT 2003.

Acta Archaeologica Academiae Scientiarum Hungaricae 70, 2019
1977.

1975.
${ }^{31}$ The original metric data providing publication: WENGER

${ }^{32}$ The original metric data providing publication: BotTYÁN 
Table 11.

Penrose distance between the male cranial series of Somogyszil-Dögkút and other comparative series (cont'd)

\begin{tabular}{|c|c|}
\hline Male cranial series & \begin{tabular}{|l} 
Penrose distance \\
(size and shape) $\mathbf{C}^{\mathrm{R} 2}$
\end{tabular} \\
\hline \multicolumn{2}{|c|}{$*=$ below the $2 \%$ error band; $* * *=$ below the $1 \%$ error band } \\
\hline Sükösd-Ságod (KŐHEGYI-MARCSIK 1971) & 0.542 \\
\hline Szebény (Tо́тн 1961) & 0.336 \\
\hline Szeged-Fehértó (LIPTÁK-VÁMOS 1969) & 0.738 \\
\hline Szeged-Kundomb (LIPTÁK-MARCSIK 1966) & 0.463 \\
\hline Szeged-Makkoserdó (VÁMOS 1973) & 0.678 \\
\hline Szekszárd-Palánk (LIPTÁK 1974) & 0.382 \\
\hline Szentes-Kaján (WENGER 1955) & 0.925 \\
\hline Tiszaderzs (based on the unpublished remeasurement data of ÉVINGER) ) $^{33}$ & 0.213* \\
\hline Tiszavárkony (LIPTÁK 1955a) & 0.727 \\
\hline Toponár 40-es őrház [Toponár, watchman's house No. 40] (based on the unpublished remeasurement data of ÉVINGER) & $0.075 * * *$ \\
\hline Üllő I. (LIPTÁK 1955b) & 0.625 \\
\hline Üllö II. (LIPTÁK 1955b) & 0.710 \\
\hline Vác-Kavicsbánya (FerENCZ 1981) & 0.334 \\
\hline Virt (HANÁKOVÁ et al 1970) & $0.194 * * *$ \\
\hline Zalakomár-Lesvári dülő II. (ÉRY 2001b) & $0.076 * * *$ \\
\hline Želovce (Zsély) (HANÁKOVÁ-STLOUKAL 1974) & $0.154 * * *$ \\
\hline Zwölfaxing (SZILVÁSSY 1980) & 0.256 \\
\hline
\end{tabular}

Roman period cemeteries of Esztergom-Bánomi dủló and Tác-Margittelep. The other series fall outside of this threshold, and thus, connect to Somogyszil-Dögkúti dủlő more loosely.

With regard to the geographical distribution, the close analogies of the late Roman period population of Somogyszil-Dögkúti dülő are all from Transdanubia, with a single exception (Tiszaderzs). With respect to the dating of these series, parallels can be found both in the Roman and in the Avar period. Viewed in a broader perspective, this indicates a continuity in the selected cranial measurements from the Roman into the Avar period on the territory of Transdanubia. On a theoretical level, this result may suggest a significant continuity of the local populations, or it may indicate that a large part of those groups that probably immigrated and settled down in the territory during the $5^{\text {th }}-8^{\text {th }}$ centuries possessed similar craniometric features as the locals had, or the combination of the two. However, with the present analysis, this question cannot be answered.

\section{REFERENCES}

ALEKSEYev-Debets 1964

BARTUCZ 1936

BARTUCZ-FARKAS 1957

BERNERT 2003

BERNERT 2005

BíRó 1999
= V. P. Alekseyev-G. F. Debets: Kraniometriya. Metodika anthropologicheskikh issledovaniy [Craniometry. Methods of Anthropological Research]. Akademiya Nauk, Moskva 1964.

= L. BARTUCZ: A Kiszombori gepida temetö koponyái (Die Gepiden-Schädel des Gräberfeldes von Kiszombor). DolgSzeged 11 (1936) 178-204.

= L. BARTUCZ-Gy. FARKAS: Zwei Adorjaner Gräberfelder der Awarenzeit aus anthropologischem Gesichtspunkte Betrachtet. ActaBiolSzeged 3 (1957) 315-347.

$=$ Zs. BERNERT: Anthropological analysis of the Avar Period cemetery of Kereki-Homokbánya (Kereki Sand pit). AnnHN 95 (2003) 125-309.

= Zs. BERNERT : Paleoantropológiai programcsomag [A software package for paleoanthropology]. Folia Anthropologica 3 (2005) 71-74.

= A. BíRó: Lesencetomaj-Piroskereszt Keszthely-kultúrás temető embertani rekonstrukciója [Anthropological examination of the Keszthely culture site of Lesencetomaj-Piroskereszt]. - Diss., ELTE Embertani Tanszék, Budapest 1999. [Manuscript]
${ }^{33}$ The original metric data providing publication: LEBZELTER 1957.
${ }^{34}$ The original metric data providing publication: WENGER

1974 
BotTYÁn 1975

BURGER 1979

CZÉKus 1985

DEZSÕ 1968

DRAVECZKY 1965

DRAVECZKY 1966

DRAVECZKY 1967

ÉRY 1966

ÉRY 1973

ÉRY 1981

ÉRY 1990

ÉRY 1992

ÉRY 1998

ÉRY 2000

ÉRY 2001a

ÉRY 2001b

ÉRY et al. 1963

ÉVINGER-BERNERT 2005

FARKAS-MARCSIK 1984

FEREMBACH et al. 1979

FERENCZ 1981

FERENC 1983

FóTHI 1988

FRÁTER 1993

GREFEN-PETERS 1987

HANÁKOVÁ-STLOUKAL 1974

HANÁKOvÁ et al. 1976

HORVÁTH et al. 2018
= O. BOTTYÁN : Pókaszepetk kora-avarkori temetőjének antropológiai értékelése (The anthropologic research of the Pókaszepetk's (SW-Transdanubia) cemetery from the early Avar Period). AnthrH 14 (1975) 5-56.

= A. Sz. BuRGER: Das spätrömische Gräberfeld von Somogyszil. FontArchHung. Akadémiai Kiadó, Budapest 1979.

= G. CzÉKUS: A moravicai (Stara Moravica) avar temető csontvázmaradványainak embertani jellemzése [Anthropological Examination of the Human Skeletal Remains from the Avar Period Cemetery of Stara Moravica]. Doktori értekezés, Szabadka 1985. [Diss., manuscript]

= Gy. DEZsỏ : Bágyogszovát avarkori népességének embertani jellemzése (An anthropological analysis of the Avar-Period population of Bágyogszovát). Arrabona 10 (1968) 79-92.

= B. DRAVECZKY: Somogyszil-Dögkúti dűlő. RégFüz I/18 (1965) 33.

= B. DRAVECZKY: Somogyszil-Dögkúti dülő. RégFüz I/19 (1966) 26.

= B. DRAVECZKY: Somogyszil. RégFüz I/20 (1967) 40-41.

= K. ÉRY: The osteological data of the 9th century population of Ártánd. AnthrH 7 (1966) 85-114.

= K. ÉRY: Anthropological data to the Late-Roman population at Pécs, Hungary. AnthrH 12 (1973) 63-114.

= K. ÉRY: Anthropologische Analyse der Population von Tokod aus dem 5. Jahrhundert. In: Die spätrömische Festung und das Gräberfeld von Tokod. Hrsg.: A. Mócsy. Akadémiai Kiadó, Budapest 1981, 223-263.

= K. ÉRY: Anthropological studies on an early Avar period population at Bačko Petrovo Selo (Yugoslavia). 2.: Analysis of the data. AnthrH 21 (1990) 33-55.

= K. ÉRY: Útmutató a csontvázleletek feldolgozásához (Posztgraduális szakképzés jegyzete) [A Guide to the Study of Human Skeletal Remains for Physical Anthropologists (For postgradual anthropology course)]. ELTE Embertani Tanszék, Budapest 1992. [Manuscript.]

$=$ K. ÉRY: Length of Limb Bones and Stature in Ancient Populations in the Carpathian Basin. Humanbiologia Budapestiensis 26. Department of Biological Department, Eötvös Loránd Univ., Budapest 1998.

= K. ÉRY: Anthropological studies on a Late Roman Period population at Tác-Margittelep. AnnHN 92 (2000) 347-453.

= K. ÉRY: Further anthropological investigations on an early Avar period population at CsákberényOrondpuszta. Alba Regia 30 (2001) 37-60.

= K. ÉRY: Késő avar kori népesség Zalakomár határából (Late Avar Period skeletal population in the vicinity of Zalakomár). ZalaiMúz 10 (2001) 141-150.

= K. ÉRY-A. KRALOVÁNSZKY-J. NEMESKÉRI: Történeti népességek rekonstrukciójának reprezentációja (A representative reconstruction of historic populations). AnthrK 7 (1963) 41-90.

$=$ S. Évinger-Zs. BERnERT: Anthropological investigation of the Avar Period cemetery of Kaposvár Road 61, Site No. 26 (Hungary). AnnHN 97 (2005) 261-319.

$=$ Gy. FARKAS-A. MARCSIK: Avar period anthropologic findings from Backa-Topola site (Yugoslavia) (Publication of data). ActaBiolSzeged 30 (1984) 191-205.

= D. Ferembach-I. SchwidetzKy-M. Stloukal: Empfehlungen für die Alters- und Geschlechtsdiagnose am Skelett. Homo 30 (1979) 1-32.

= M. FERENCZ: Some data to the paleoanthropology of the Avar Period's population in Hungary. AnthrH 17 (1981) 23-67.

= M. FERENCZ: The Avar-age cemetery at Solymár. AnthrH 18 (1983) 9-41.

= E. FóтнI: The anthropological investigation of the Avar-age cemetery of Fészerlak. AnthrH 20 (1988) 31-53.

= E. FRÁTER: Embertani és szerológiai vizsgálatok Budapest III. Kaszás dülő, Raktárrét római kori népességén (Anthropological and serological studies on a Roman Period population at Kaszás dülö, Raktárrét, Budapest). AnthrK 35 (1993) 39-60.

= S. GREFEN-PETERS: Anthropologische und zoologische Auswertung. In: Das awarische Gräberfeld von Leobersdorf, Nö. Hrsg.: F. Daim. Verlag der ÖAW, Wien 1987, 79-323.

= H. HANÁKOVÁ-M. STLOUKAL: Antropologický výzkum pohřebiště ze 7.-8. století v Želovcích (Anthropologische Erforschung des Gräberfeldes aus dem 7.-8. Jahrhundert in Želovce). S1A 22/1 (1974) 129-188.

= H. HANÁKovÁ-M. STloukaL-L. VYhnANEK: Kostry ze slovansko-avarského pohřebiště ve Virtu [Human skeletal remains from the Slavic-Avar cemetery of Virt]. SbNM 32/2-4 (1976) 57-113.

= F. Horváth-A. MihácZi-PÁlfi-S. Évinger-Zs. Bernert: Barbarisierte Römer - Romanisierte Barbaren? Interpretationsmöglichkeiten der fremden Komponente am Beispiel des Gräberfeldes von Somogyszil. Antaeus 35-36 (2018) 39-65. 
HuSZÁR-SCHRANZ 1952

IsCAN et al. 1984

KÖHEGYI-MARCSIK 197 LAMENDIN et al. 1992

LEBZELTER 1957

LIPTÁK 1954

LIPTÁK 1955a

LIPTÁK $1955 b$

LIPTÁK 1974

LIPTÁK 1983

LIPTÁK-MARCSIK 1966

LIPTÁK-MARCSIK 1976

LIPTÁK-VÁMOS 1969

MARTIN-SALLER 1957

MEINDL-LOVEJOY 1985

MERCZI 2001

MerCZi 2008

NEMESKÉRI et al. 1960

ORTNER 2003

PenRose 1952

PODANI 1997

RÖSING-SCHWIDETZKY 1977

SCHINZ et al. 1952

SCHOUR-MASSLER 1941

SCHWIDETZKY 1967

SJøVOLD 1990

SusKOVICS 1993

STLOUKAL-HANÁKOVÁ 1978

SZILVÁSSY 1980
= GY. HusZÁR-D. SchranZ: A fogszuvasodás elterjedése a Dunántúlon, az újkőkortól az újkorig [The frequency of (dental) caries in Transdanubia from the Neolithic to the Modern Age]. Fogorvosi Szemle 45 (1952) 3-38.

= M. Y. IsCAN-S. Loth-R. WRIGHT : Age estimation from the rib by phase analisys: White males. Journal of Forensic Sciences 29 (1984) 1094-1104.

= M. KöHegYI-A. MARCSIK: The Avar-Age cemetery at Sükösd. AASzeged 14 (1971) 87-94.

= H. LAmendin-E. Baccino- J. F. Humbert-J. C. TAvernier-R. M. Nossintchouk-A. Zerilli: A simple technique for age estimation in adult corpses: The two criteria dental method. Journal of Forensic Sciences 37/5 (1992) 1373-1379.

= V. LEBZELTER: Beschreibung der Skelettreste von Tiszaderzs. CraHung 2 (1957) 3-59.

= P. LIPTÁK: Les Avares des environs de Kecel. BiolKözl 2 (1954) 159-180.

= P. LIPTÁK: A Duna-Tisza köze antropológiájának főbb kérdései a VII-VIII. században [Major Questions of Anthropology Concerning the $6^{\text {th }}-8^{\text {th }}$ Century Populations of the Danube-Tisza Interfluve]. Diss, Budapest 1955. [Manuscript]

= P. LIPTÁK: Recherches anthropologiques sur les ossements avares des environs d'Üllő. ActaArchHung 6 (1955) 231-316.

= P. LIPTÁK: Anthropological analysis of the Avar-Period population of Szekszárd-Palánkpuszta. ActaBiolSzeged 20 (1974) 199-211.

= P. LIPTÁK: Avars and Ancient Hungarians. Akadémiai Kiadó, Budapest 1983

= P. LIPTÁK-A. B. MARCsiK: Szeged-Kundomb avar kori népességének embertani vizsgálata (Die anthropolische Untersuchung des Gräberfeldes Szeged-Kundomb aus der Awarenperiode). AnthrK 10 (1966) 13-56

= P. LIPTÁK-A. MARCSIK A Madaras-Téglavető melletti avar temető csontvázmaradványainak embertani jellemzése (Anthropologische Charakteristik der Skelettreste aus dem awarischen Gräberfeld bei Madaras-Téglavető). Cumania 4 (1976) 115-140.

= P. LIPTÁK-K. VÁMOS: A "Fehértó-A" megnevezésű avar kori temető csontvázanyagának embertani vizsgálata (Anthropolische Untersuchung des Skelettmaterial des awarenzeitlichen Gräberfeldes von „Fehértó-A”). AnthrK 13 (1969) 3-30.

= R. Martin-K. SAlleR: Lehrbuch der Antropologie. I. Gustav Fischer Verlag, Stuttgart 1957.

$=$ R. S. MEINDL-C. O. LoveJoY: Ectocranial suture closure: A revised method for the determination of skeletal age at death based on the lateral-anterior sutures. AJPA 68 (1985) 57-66.

= M. MERCZI: Embertani adatok a Dunakanyar (Visegrád-Diós) késő római kori népességéhez (Anthropological data to the Late Roman Period population of the Danube Bend - Visegrád-Diós). AnthrK 42 (2001) 33-44.

= M. MerCZI (2008): A Castellumtemető (Esztergom-Bánomi dűlő) népességének embertani vizsgálata - Anthropologische Analyse der Bevölkerung des Kastellgräberfeldes in Esztergom-Bánomer Flur. In: H. M. Kelemen: Esztergom későrómai temetői - Die Spätrömischen Gräberfelder von Esztergom. Libelli archaeologici SerN 3. Magyar Nemzeti Múzeum, Budapest 2008, 403-478.

= J. Nemeskéri-L. HARsÁNYI-Gy. AcsÁdi: Methoden zur Diagnose des Lebensalters von Skelettfunden. Anthropologischer Anzeiger 24 (1960) 103-115.

= D. J. OrTnER : Identification of Pathological Conditions in Human Skeletal Remains. Academic Press, Amsterdam, etc. 2003. $-2^{\text {nd }}$ ed.

= L. S. Penrose: Distance, size and shape. Annals of Eugenics 18 (1952) 337-343.

= J. PODANI: Bevezetés a többváltozós biológiai adatfeltárás rejtelmeibe avagy „Mit kezdjünk azzal a rengeteg adattal?" [An Introduction to the Multivariate Statistical Analyses used in Biological Research - "What can we do with those myriads of data?" ]. Scientia Kiadó, Budapest 1997.

= F. W. RösING-I. SchwidETZKY: Vergleichend-statistische Untersuchungen zur Anthropologie des frühen Mittelalters (500-1000 n.d.Z.). Homo 28 (1977) 65-115.

$=$ H. SchinZ-W. BAENSCH-E. FriedL-E. Uehlinger: Ossifikationstabelle. In: Lehrbuch der RöntgenDiagnostik. 1-4. Hrsg.: H. Schinz. Thieme, Stuttgart 1952. - 5. Aufl.

= J. Schour-M. MASSLER: The development of the human dentation. Journal of American Dental Association 28 (1941) 1153-1160.

= I. SCHwIDETZKy: Erfahrungen mit dem Penrose-Abstand. Homo 18/3 (1967) 140-144.

$=\mathrm{T}$. SıøVOLD : Estimation of stature from long bones utilizing the line of organic correlation. Human Evolution 5 (1990) 431-447.

= Cs. SusKoviCs: Siófok-Kiliti avar kori népességének embertani vázlata (Anthropological evaluation of the population of Siófok-Kiliti living in the Avar Period). AnthrK 35 (1993) 61-81.

= M. STlouKAL-H. HANÁKOvÁ: Die Länge der Längsknochen altslawischer Bevölkerungen - Unter besonderer Berücksichtigung von Washstumsfragen. Homo 29 (1978) 53-69.

= J. SzILVÁssy: Die Skelette aus dem awarischen Gräberfeld von Zwölfaxing in Niederösterreich. Anthropologische Forschungen 3. Wien 1980. 
RENDES-TÓTH 2000

Thома 1978

ThURZO 1984

ToDD 1920

TÓTH 1961

UBELAKER 1989

VARGA et al. 2003

VARGA et al. 2005

VÁMOS 1973

WEDEL-GaLloway 2014

WENGER 1953

WENGER 1955

WENGER 1957

WENGER 1966

WENGER 1968

WENGER 1974

WENGER 1975

WENGER 1977
= K. T. RENDES-G. TóтH: Gyenesdiás avar kori népességének kraniometriai értékelése (Craniometric evaluation of the Avar Period population of Gyenesdiás). - In: A népvándorláskor kutatóinak kilencedik konferenciája : Eger, 1998. szeptember 18-20. Eds: T. Petercsák, A. Váradi. Heves megyei régészeti közlemények 2. Eger 2000, 467-472.

= A. Thoma: Distance et forma entre groupes. Bulletins et Mémoires de la Société d'Anthropologie de Paris 5/13 (1978) 15-22.

= M. Thurzo: Metrische Merkmale der menschlichen Skelettreste aus dem slawisch-awarischen Gräberfeld (7.-9. Jh. u.Z.) in Košice-Sebastovce (bez. Košice-Stadt), Ostslowakei. AInA 3 (1984) $1-262$.

$=\mathrm{T}$. W. ToDD: Age changes in the pubis bone. I.: The male white pubis. American Journal of Physical Anthropology 3 (1920) 285-334.

$=$ T. TóTH: The cemetery of Szebény I ( $8^{\text {th }}$ century) from the Avar epoch. AnnHN 53 (1961) 571-613.

= D. H.Ubelaker: Human Skeletal Remains, Excavation, Analysis, Interpretation. Taraxacum, Washington 1989.

= P. VArgA-Zs. Bernert-Gy. Gyenis-E. Fóthi: Multivariate statistics on Roman and Migration Period populations of the Carpathian Basin. Anthropologie 16/1-2 (2003) 135-144.

= P.,VARGA-Zs. BERNERT-E. FóTHI: Antropológiai adatok a Keszthely-Dobogó római kori temetőhöz [Some anthropological data to the research of the Roman Period cemetery of Keszthely-Dobogó]. In: IV. Kárpát-medencei Biológiai Szimpozium, 2005. október 17-19. Előadáskötet. Ed.: Z. Korsós. Budapest 2005, 193-196.

= K. VÁMOS: „Szeged-Makkoserdő” avar kori népességének embertani vizsgálata (Die anthropolische Untersuchung der awarenzeitlichen Bevölkerung von „Szeged-Makkoserdo””). AnthK 17 (1973) 29-39.

= V. L. WedEL-A. GALlowAY (eds): Broken Bones. Anthropological Analysis of Blunt Force Trauma. Thomas Books, Springfield, IL 2014. $-2^{\text {nd }}$ ed.

= S. WENGER: L'anthropologie du cimetiere de Jánoshida-Tótkérpuszta. AnnHN 4 (1953) 231-244.

= S. WENGER: Szentes-Kaján népvándorlás kori népességének embertani típusai (VII.-VIII. század) (Types anthropologiques de Szentes-Kaján provenant du VII $-\mathrm{VIII}^{\mathrm{e}}$ siècles). AnnHN 6 (1955) 391-410.

= S. Wenger: Données ostéométriques sur le matériel anthropologiques du cimetiere d'AlattyánTulát, provenant de l'époque avare. CraHung 2 (1957) 1-55.

= S. Wenger: Anthropologie de la population d'Elöszállás-Bajcsihegy provenant des temps avar. AnthrH 7 (1966) 115-206.

$=\mathrm{S}$. Wenger: Data to the anthropology of the Avar Period population of the Transdanubia (The anthropology of the Avar Period cemetery at Kékesd). AnthrH 8 (1968): 59-96.

$=\mathrm{S}$. WeNGER: Déldunántúl avarkori népességének embertani problémái (On the anthropological problems of the Avar Age populations in the Southern Transdanubia). AnthrH 13 (1974) 5-86.

= S. Wenger Paleoanthropology of population deriving from the Avar Period at Fészerlak-puszta. AnthrH 14 (1975) 57-110.

$=\mathrm{S}$. WENGER Analyses anthropologiques de nouvelles découvertes de Keszthely (Transdanubie) provenant de l'époque avare. AnthrH 15 (1977) 125-190.

Open Access. This is an open-access article distributed under the terms of the Creative Commons Attribution 4.0 International License (https://creativecommons.org/licenses/ by/4.0), which permits unrestricted use, distribution, and reproduction in any medium, provided the original author and source are credited, a link to the CC License is provided, and changes - if any - are indicated. (SID_1) 